\title{
Activation of sirtuin 1 as therapy for the peroxisomal disease adrenoleukodystrophy
}

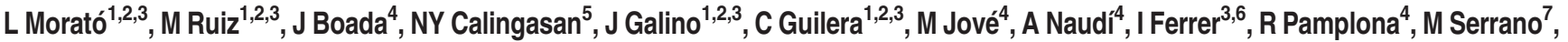 \\ M Portero-Otín ${ }^{4}$, MF Beal ${ }^{5}$, S Fourcade ${ }^{1,2,3}$ and A Pujol ${ }^{*, 1,2,3,8}$
}

Oxidative stress and mitochondrial failure are prominent factors in the axonal degeneration process. In this study, we demonstrate that sirtuin 1 (SIRT1), a key regulator of the mitochondrial function, is impaired in the axonopathy and peroxisomal disease X-linked adrenoleukodystrophy (X-ALD). We have restored SIRT1 activity using a dual strategy of resveratrol treatment or by the moderate transgenic overexpression of SIRT1 in a X-ALD mouse model. Both strategies normalized redox homeostasis, mitochondrial respiration, bioenergetic failure, axonal degeneration and associated locomotor disabilities in the X-ALD mice. These results indicate that the reactivation of SIRT1 may be a valuable strategy to treat X-ALD and other axonopathies in which the control of redox and energetic homeostasis is impaired.

Cell Death and Differentiation (2015) 22, 1742-1753; doi:10.1038/cdd.2015.20; published online 27 March 2015

Sirtuins are highly conserved $\mathrm{NAD}^{+}$-dependent deacetylases with a critical impact on metabolic adaptive responses. In mammals, among the seven members of the sirtuin family (SIRT1-SIRT7), SIRT1 has been the most extensively characterized. ${ }^{1}$ SIRT1 promotes the generation of new mitochondria through the activation of peroxisome proliferator-activated receptor gamma coactivator 1 alpha (PGC-1a), which orchestrates the mitochondrial biogenesis program through the transcriptional activation of nuclear respiratory factors (NRF-1 and NRF-2) and mitochondrial transcription factor A (TFAM). ${ }^{2}$ SIRT1 activity is finely regulated not only by gene expression and protein levels but also by posttranslational modifications, its oligomeric status, the interaction with the $\mathrm{DBC} 1$ and AROS proteins, and the levels of $\mathrm{NAD}^{+} / \mathrm{NADH} / \mathrm{NAM}^{3}{ }^{3}$ In addition, SIRT1 activity is modulated by several synthetic or natural molecules such as the polyphenol resveratrol $\left(3,4^{\prime}, 5\right.$-trihydroxystilbene: RSV). ${ }^{4}$ The activation of SIRT1 by transgenic overexpression or pharmacologically with RSV has been shown to prevent the pathology in diseases commonly associated with mitochondrial dysfunction such as the metabolic syndrome and neurodegenerative disorders, ${ }^{1,5,6}$ albeit recent findings cast doubts on the universal validity of the results. ${ }^{7,8}$ Although prominent axonal pathology often precedes cell body loss in many neurodegenerative diseases, ${ }^{9}$ the potential of SIRT1 activation in axonal degeneration has not been addressed in depth. In this work, we take advantage of the cellular and animal models of the rare monogenic neurodegenerative disease named X-linked adrenoleukodystrophy (X-ALD, (McKusick No.300100)) to evaluate the impact of SIRT1 function on axonal degeneration. X-ALD is the most prevalent peroxisomal disorder (minimum incidence 1:17000 newborns), characterized by brain inflammatory demyelination and/or axonopathy of long tracts in spinal cords, adrenal insufficiency and a pathognomonic accumulation of very longchain fatty acids (VLCFA) in plasma and tissues, in particular hexacosanoic acid (C26:0). ${ }^{10,11}$ The disease phenotypes range from adrenal insufficiency only (Addison's only) to severe inflammatory cerebral demyelination, of either childhood or adult onset (CCALD or CAMN respectively), which advances rapidly to dementia and death. Most frequently, adult patients show mainly a slowly progressive spastic paraparesis without cerebral inflammatory demyelination, peripheral neuropathy and sphincter disturbances, the

\footnotetext{
${ }^{1}$ Neurometabolic Diseases Laboratory, Bellvitge Biomedical Research Institute (IDIBELL), L'Hospitalet de Llobregat, 08908 Barcelona, Spain; ${ }^{2}$ Center for Biomedical Research on Rare Diseases (CIBERER) ISCIII, Spain; ${ }^{3}$ Institute of Neuropathology, University of Barcelona, L'Hospitalet de Llobregat, 08908 Barcelona, Spain; ${ }^{4}$ Experimental Medicine Department, University of Lleida-IRBLleida, 25008 Lleida, Spain; ${ }^{5}$ Department of Neurology and Neuroscience, Weill Cornell Medical College, 1006 New York, USA; ${ }^{6}$ Center for Biomedical Research on Neurodegenerative Diseases (CIBERNED) ISCIII, Spain; ${ }^{7}$ Tumor Suppression Group, Spanish National Cancer Research Centre (CNIO), 28029 Madrid, Spain and ${ }^{8}$ Catalan Institution of Research and Advanced Studies (ICREA), Barcelona, Spain

${ }^{*}$ Corresponding author: A Pujol, Neurometabolic Diseases Laboratory, IDIBELL, Hospital Duran i Reynals, Gran Via 199, L'Hospitalet de Llobregat, 08908 Barcelona, Spain. Tel: +34 932607343; Fax: +34 932607414. E-mail: apujol@idibell.cat

Abbreviations: AD, Alzheimer's disease; AMN, adrenomyeloneuropathy; APP, amyloid precursor protein; ATP, adenosine triphosphate; C26:0, hexacosanoic acid; CCALD, childhood cerebral adrenoleukodystrophy; CAMN, cerebral adrenomyeloneuropathy; CTL, control; ETC, electron transport chain; FOXO1, forkhead box protein 01; GFAP, glial fibrillary acidic protein; GR, glutathione reductase; GSH, reduced glutathione; GSSG, glutathione disulfide; HD, Huntington's disease; HSP, Hereditary Spastic Paraplegia; MDA, malondialdehyde; mPTP, mitochondrial permeability transition pore; mtDNA, mitochondrial DNA; NAD ${ }^{+}$, nicotinamide adenine dinucleotide (oxidized form); NADH, nicotinamide adenine dinucleotide (reduced form); NAM, nicotinamide; nDNA, nuclear DNA; NDUFB8, NADH dehydrogenase (ubiquinone) 1 beta subcomplex, 8, 19kDa; NMAT1, nicotinamide mononucleotide adenylyl transferase1; NRF-1, nuclear respiratory factor-1; NRF-2, nuclear respiratory factor-2; OXPHOS, oxidative phosphorylation; PD, Parkinson's disease; PGC-1 $\alpha$, peroxisome proliferator-activated receptor gamma coactivators-1 alpha; PK, pyruvate kinase; ROS, reactive oxygen species; RSV, resveratrol; RT-PCR, real time PCR; SDHB, succinate dehydrogenase complex, subunit B, iron sulfur (Ip); SIRT1, sirtuin 1; SMI32, neurofilament $\mathrm{H}$ non-phosphorylated; SPG7, spastic paraplegia 7; TFAM, mitochondrial transcription factor A; VDAC1, voltage-dependent anion channel 1; VLCFA, very long-chain fatty acids; WIds, Wallerian degeneration slow; X-ALD, X-linked adrenoleukodystrophy; WT, wild type; $\Delta \Psi \mathrm{m}$, inner mitochondrial membrane potential
}

Received 10.8.14; revised 16.12.14; accepted 29.1.15; Edited by N Bazan; published online 27.3.15 
disease manifestations typical of adrenomyeloneuropathy (AMN) of male, and many women carriers. ${ }^{11,12}$ In all its variants, the disease is caused by the loss of function of the $A B C D 1$ gene in Xq2.8, which encodes a transmembrane transporter involved in the import of VLCFA and VLCFACoenzyme A esters into the peroxisome for their breakdown by beta-oxidation. ${ }^{13}$ Lack of phenotype-genotype correlations within the same nuclear family contribute to the enigmatic nature of the disease. Therapeutic options remain scarce, with a small window of opportunity to apply allogeneic bone marrow transplant or new gene therapy for CCALD. ${ }^{14}$ No therapeutic options are currently available for AMN. ${ }^{15}$ The classical inactivation of $A b c d 1$ in mice $\left(A b c d 1^{-}\right)$results in the accumulation of VLCFA in target organs such as the central nervous system and adrenals. ${ }^{16,17,18}$ These animals present a late onset axonopathy in the spinal cord, in the absence of inflammatory demyelination in the brain, resembling the spastic paraparesis and lower extremity weakness of adrenomyeloneuropathy (AMN). ${ }^{18,19}$ We previously reported oxidative damage in spinal cords from presymptomatic Abcd1- mice, ${ }^{20-22}$ together with metabolic failure, ${ }^{23}$ disrupted mitochondrial biogenesis ${ }^{24}$ and impaired mitochondrial oxidative phosphorylation and function. ${ }^{25-27}$ Evidences for oxidative damage in X-ALD patients are also found in brain $^{28}$ and blood samples. ${ }^{29-31}$

In this study. we demonstrate that the promotion of mitochondrial function through SIRT1 activation emerges as a valuable therapeutic option to prevent axonal demise in X-ALD.

\section{Results}

Dysregulation of SIRT1 in X-ALD. To ascertain whether SIRT1 is involved in the axonal degeneration process in $X$-ALD, we used the Abcd1-deficient mouse, a model for lateonset X-ALD or adrenomyeloneuropathy (AMN), which presents chronic and slowly progressing axonopathy of spinal cords. We measured SIRT1 levels in the spinal cord of 12-month-old $\mathrm{Abcd}^{-}$mice, 6 months prior to the disease onset (Figures 1a and c). We observed that SIRT1 (mRNA, protein, activity) is downregulated in the spinal cord of $\mathrm{Abcd1}^{-}$mice (Figures $1 \mathrm{a}$ and $\mathrm{c}$ ). Moreover, we detected decreased levels of SIRT1 in the affected white matter of $X$-ALD patients (Figure 1d). These findings are in agreement with the alteration in the PGC-1a-induced mitochondrial biogenesis reported recently in X-ALD mice and patients. ${ }^{24}$

RSV prevents ROS generation and protects mitochondrial function in X-ALD fibroblasts. Aiming at translational opportunity, we investigated a putative beneficial effect of SIRT1 activation in X-ALD. We treated fibroblasts from control and X-ALD patients with RSV, a SIRT1 activator widely used as a dietary supplement and also in clinical trials for several metabolic conditions. ${ }^{32}$ We formerly reported that an excess of $\mathrm{C} 26: 0$, the biochemical hallmark and metabolite accumulated in X-ALD, triggers mitochondrial ROS production from the electron transport chain (ETC) complexes in human fibroblasts. ${ }^{25}$ Interestingly, RSV treatment prevented C26:0-dependent ROS generation in these cells (Figure 2a). Owing to their underlying mitochondrial frailty, fibroblasts from X-ALD patients die when they are forced to rely on mitochondrial metabolism to obtain energy, as revealed by culturing in a glucose-free galactose-enriched medium. Galactose-induced cell death is dependent on the mitochondrial permeability transition pore (mPTP) opening and is preceded by an increase in ROS generation, a drop in ATP production and a decrease in the inner mitochondrial membrane potential $(\Delta \psi \mathrm{m}){ }^{26}$ We thus tested the potential of RSV to prevent galactose-induced cell demise. Remarkably, RSV treatment prevented cell death (Figure $2 b$ ) and normalized ATP levels (Figure 2c) and the $\Delta \Psi \mathrm{m}$ (Figure 2d) together with a decrease in ROS production in the presence of galactose (Figure 2e). To investigate whether this protective mechanism of RSV was SIRT1-dependent in our in vitro model, the cells were incubated with RSV together with EX-527, a potent SIRT1 inhibitor. ${ }^{33}$ We observed that the ability of RSV to neutralize both C26:0- (Figure 2a) and galactose-induced ROS (Figure 2e) was SIRT1-independent, suggesting that RSV may act as a ROS scavenger at the doses used in this system. In contrast, EX-527 partially abolished the ability of RSV to prevent cell death (Figure $2 b$ ) and completely abolished the positive effects of RSV on the ATP content (Figure $2 \mathrm{c}$ ) and $\Delta \Psi_{\mathrm{m}}$ (Figure $2 \mathrm{~d}$ ), indicating that mitochondrial function is dependent on SIRT1 activity. a

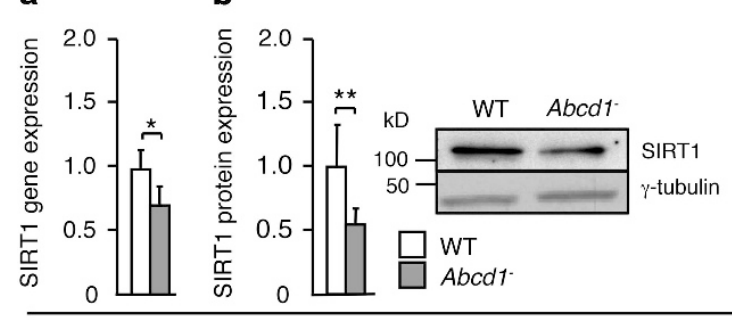

C

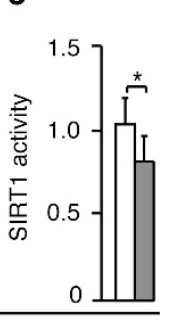

d

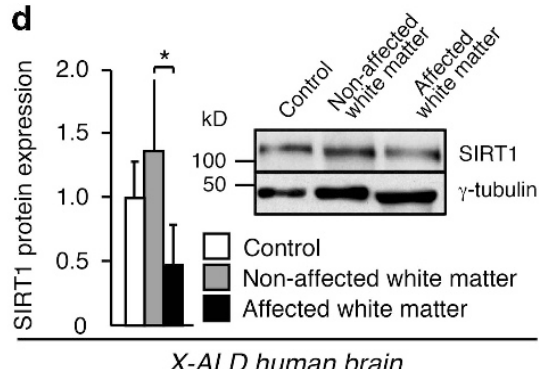

Figure 1 SIRT1 dysregulation in X-ALD. (a-c) SIRT1 analysis in spinal cord of 12-month-old WT $(n=4)$ and Abcd1 ${ }^{-}$mice $(n=6)$. (a) SIRT1 gene expression analyzed by quantitative RT-PCR. Relative expression values were normalized to WT mice. (b) SIRT1 protein levels. Representative immunoblot is shown. Protein levels are normalized relative to $\gamma$-tubulin and quantification is represented as fold change to WT mice. (c) SIRT1 activity. Relative activity values were normalized to WT mice. (d) Representative immunoblot for SIRT1 in control $(n=4)$ and in non-affected $(n=4)$ and affected white matter from X-ALD patients $(n=4)$. Protein levels are normalized relative to $\gamma$-tubulin and quantification is represented as fold change with respect to control white matter. Values are expressed as mean \pm S.D. $\left({ }^{*} P<0.05,{ }^{* *} P<0.01,{ }^{* * *} P<0.001\right.$, unpaired $t$ test (panels a-c)) or one way-ANOVA followed by $t$ test post hoc (paired $t$ test between non-affected and affected white matter and unpaired $t$ test between control and non-affected white matter and between control and affected white matter, panel $\mathbf{d})$ 
a

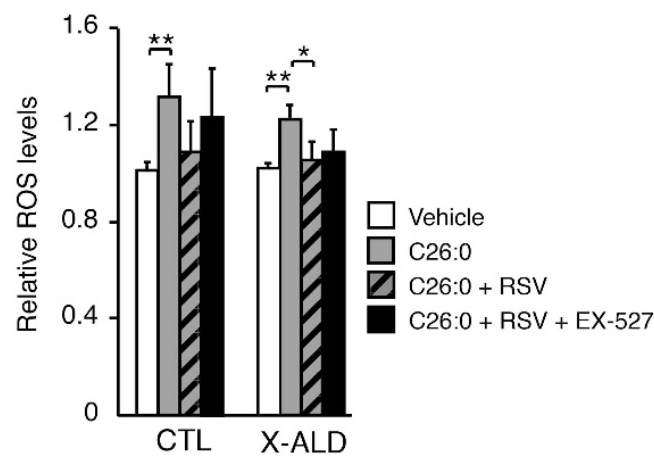

b

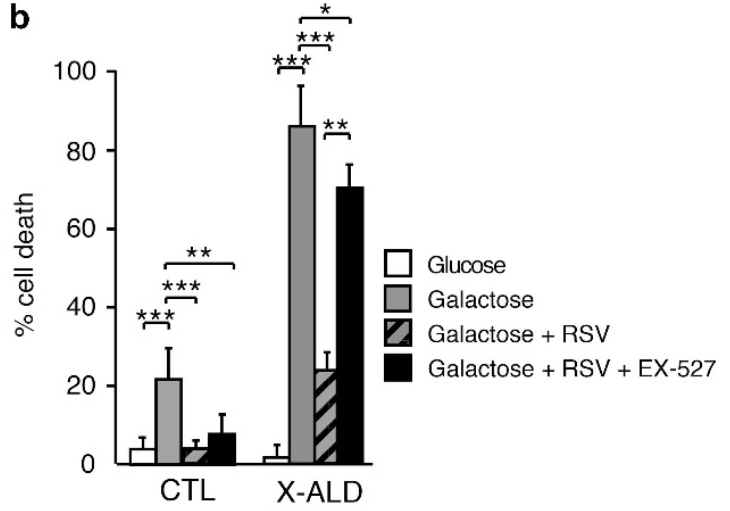

e

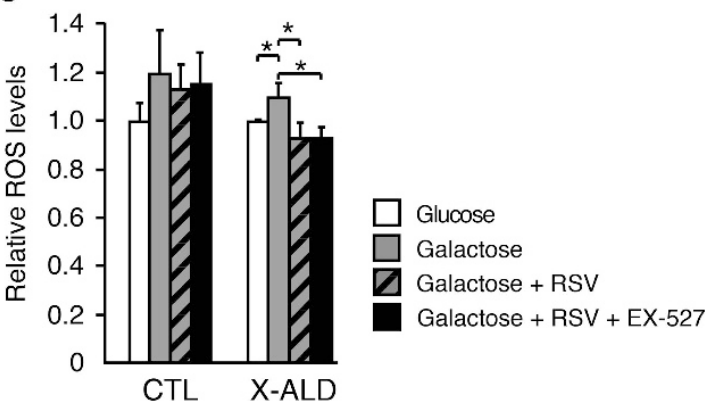

Figure 2 RSV prevents ROS production and mitochondrial dysfunction through SIRT1-dependent and -independent mechanisms in X-ALD fibroblast. (a) ROS production measured in control (CTL) and X-ALD fibroblasts treated with $50 \mu \mathrm{M}$ of C26:0, $25 \mu \mathrm{M}$ of RSV and $10 \mu \mathrm{M}$ of EX-527 for $24 \mathrm{~h}$. ROS values were normalized to untreated cells ( $n=6$ per genotype and condition). (b) Galactose-induced cell death in CTL and X-ALD fibroblasts treated with $25 \mu \mathrm{M}$ of RSV and $10 \mu \mathrm{M}$ of EX-527 for $150 \mathrm{~h}$ ( $n=6 \mathrm{per}$ genotype and condition). (c) ATP content in CTL and X-ALD fibroblasts treated with $25 \mu \mathrm{M}$ of RSV and $10 \mu \mathrm{M}$ of EX-527 for $136 \mathrm{~h}$ ( $n=6$ per genotype and condition). (d) Percentage of cells with inner mitochondrial membrane depolarization in CTL and X-ALD fibroblasts treated with $25 \mu \mathrm{M}$ of RSV and $10 \mu \mathrm{M}$ of EX-527 for $136 \mathrm{~h}$ ( $n=6$ per genotype and condition). (e) ROS production in CTL and X-ALD fibroblasts treated with $25 \mu \mathrm{M}$ of RSV and $10 \mu \mathrm{M}$ of EX-527 for $136 \mathrm{~h}$ ( $n=6$ per genotype and condition). ROS values were normalized to untreated cells. Values are expressed as mean \pm S.D. $\left({ }^{\star} P<0.05,{ }^{* \star} P<0.01\right.$ and ${ }^{* \star *} P<0.001$, one-way ANOVA followed by Tukey's HSD post hoc)

Thus, RSV exerted its actions through a combination of SIRT1-dependent and -independent beneficial effects in the $\mathrm{X}$-ALD in vitro model.

The activation of SIRT1 in Abcd1- mice rescues mitochondrial biogenesis. Given the rich debate generated over the past years regarding the capacity of RSV to directly or indirectly activate SIRT1 and other off-target effects of resveratrol, ${ }^{34}$ we pursued a dual strategy aimed at increasing SIRT1 function either directly by transgenic overexpression or pharmacologically using RSV. Therefore, we used two independent cohorts of animals. The first group comprised WT mice, $A b c d 1^{-}$mice and Abcd1- mice crossed to a mouse model showing moderate and ubiquitous transgenic overexpression of SIRT1 (SIRT1Tg), driven by its own promoter in the BAC genomic region, ${ }^{35}$ and then generating the $\mathrm{Abcd1}^{-}$/ SIRT1Tg model. The second independent group of animals included WT mice, $A b c d 1^{-}$mice and $A b c d 1^{-}$mice fed for 7 weeks with RSV up to the age of 12 months ( $\left.A b c d 1^{-}+\mathrm{RSV}\right)$. Both $A b c d 1^{-} / S I R T 1 T g$ and $A b c d 1^{-}+$RSV mice at 12 months old showed a normalization of SIRT1 activity, whereas only Abcd1/SIRT1Tg mice exhibited a two-fold increase in SIRT1 protein levels in the spinal cord (Supplementary Figure S1).

Notably, we observed that SIRT1 activation in either model normalized mitochondrial biogenesis based on several parameters: (i) the mitochondrial DNA to nuclear DNA (mtDNA/
nDNA) ratio (Figures 3a and d); (ii) the levels of SIRT1, NRF1 and TFAM mRNA (Figures $3 \mathrm{~b}$ and e); (iii) and the protein levels of NDUFB8 and SDHB, subunits of the ETC (Figures $3 \mathrm{c}$ and $\mathrm{f}$ ).

The activation of SIRT1 in $\mathrm{Abcd1}^{-}$mice prevents energetic failure and increases antioxidant defense. Energy and redox homeostasis are disrupted in presymptomatic $\mathrm{Abcd1}^{-}$mice. At 12 months of age, these mutant mice show lower levels of $\mathrm{ATP}$, increased $\mathrm{NAD}^{+} / \mathrm{NADH}$ ratio and decreased activity and protein levels of pyruvate kinase (PK), a key regulator of glycolysis, in the spinal cord. Redox imbalance is reflected by diminished amounts of reduced glutathione (GSH), the primary antioxidant of the cell, and by a decreased activity of glutathione reductase (GR), the enzyme that reduces glutathione disulfide (GSSG) to $\mathrm{GSH}^{23,24}$ In $\mathrm{Abcd1}^{-}$mice, both the overexpression of SIRT1 and the treatment with RSV prevented the drop in ATP levels (Figures $4 \mathrm{a}$ and $\mathrm{b}$ ) and normalized the $\mathrm{NAD}^{+} / \mathrm{NADH}$ ratio (Figures $4 \mathrm{c}$ and $\mathrm{d}$ ), as well as the PK activity (Figures $4 \mathrm{e}, \mathrm{h}$ and Supplementary Figure S2). Moreover, GSH levels (Figures $4 \mathrm{f}$ and i) and GR activity (Figures $4 \mathrm{~g}$ and $\mathrm{j}$ ) were normalized after SIRT1 activation, suggesting that SIRT1 regulates the antioxidant defense in vivo. These data are supported by the reduced levels of lipooxidation in $A b c d 1^{-} /$ Abcd2 $2^{-/} /$SIRT1Tg and $A b c d 1^{-} / A b c d 2^{-/}+$RSV mice (Figures $\left.6 c^{\prime}-f^{\prime}\right)$. Finally, our results led us to conclude that the 
a

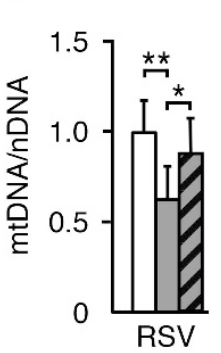

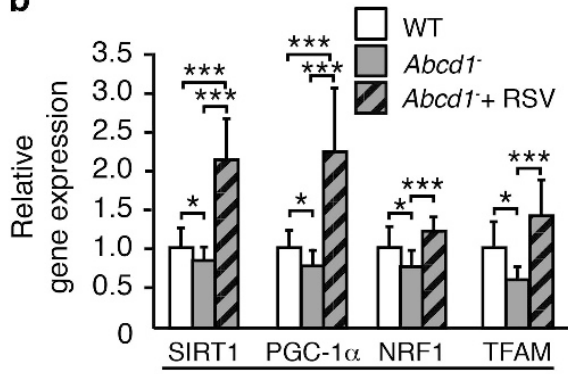

RSV
C

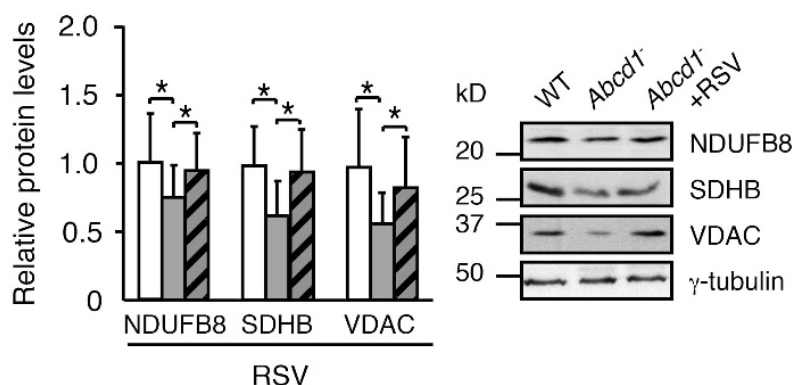

f

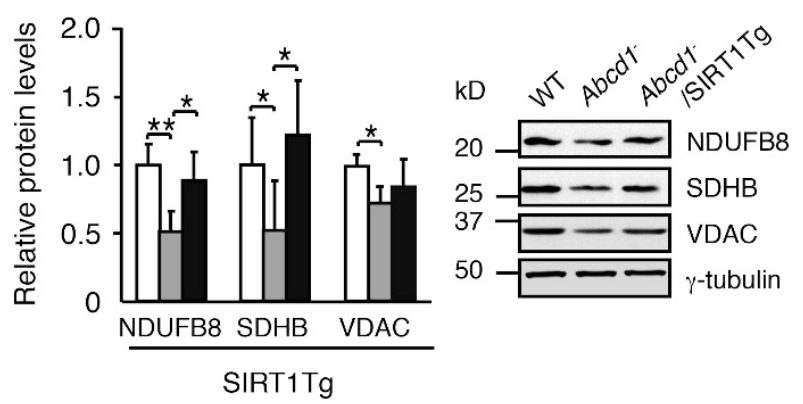

Figure 3 Activation of SIRT1 rescues mitochondrial depletion in $A b c d 1^{-}$mice. (a and d) mtDNA content analyzed by quantitative RT-PCR and expressed as the ratio of mtDNA to nDNA (mtDNA/nDNA) in (a) the spinal cord of 12-month-old WT $(n=5), A b c d 1^{-}(n=6)$ and $A b c d 1^{-}+$RSV mice $(n=6)$ and in (d) the spinal cord of 12-month-old WT $(n=5), A b c d 1^{-}(n=6)$ and $A b c d 1^{-} / S I R T 1 T g$ mice $(n=6)$. Relative expression values were normalized to WT mice. (b and e) SIRT1, PGC- $1 \alpha$, NRF1 and TFAM relative gene expression analyzed by quantitative RT-PCR in (b) the spinal cord of 12-month-old WT $(n=5)$, Abcd1- $(n=6)$ and Abcd1 ${ }^{-}+$RSV mice $(n=6)$ and in (e) 12-month-old WT $(n=5), A b c d 1^{-}(n=6)$ and $A b c d 1^{-} / S I R T 1 T g$ mice $(n=6)$. Relative expression values were normalized to WT mice. (c and f) NDUFB8, SDHB and VDAC protein expression in (c) the spinal cord of 12-month-old WT $(n=5), A b c d 1^{-}(n=6)$ and $A b c d 1^{-}+$RSV mice $(n=6)$ and in (f) 12-month-old WT $(n=5), A b c d 1^{-}(n=6)$ and Abcd1-/SIRT1Tg mice $(n=6)$. Representative immunoblots are shown. Protein levels are normalized respect to $\gamma$-tubulin and quantification is represented as fold change respect to WT mice. Values are expressed as mean \pm S.D. $\left({ }^{\star} P<0.05,{ }^{* \star} P<0.01\right.$ and ${ }^{* \star *} P<0.001$, one-way ANOVA followed by Tukey's HSD post hoc)

activation of SIRT1 activation in $A b c d 1^{-}$mice prevents oxidative stress, which replenishes the pool of GSH and decreases lipid peroxide levels.

The activation of SIRT1 promotes mitochondrial function in $\boldsymbol{A b c d 1}^{-}$mice. We sought to investigate the effects of restoring SIRT1 activity in preserving mitochondrial function. Using high-resolution respirometry (Oroboros) analysis in freshly sectioned spinal cord slices, we revealed that activation of SIRT1 either with RSV or with transgenic overexpression of SIRT1 prevents the impaired mitochondrial oxidative phosphorylation previously observed in 12-monthold $A b c d 1^{-}$mice. ${ }^{25}$ This ex vivo approach allowed us to demonstrate that SIRT1 activation normalizes the mitochondrial content as shown in Figure 3 and also preserves mitochondrial respiration in the spinal cord of $A b c d 1^{-}$mice (Figures 4k and I).

The activation of SIRT1 normalizes locomotor deficits in

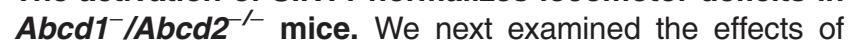
SIRT1 activation in the axonopathy and associated locomotor disability exhibited in the X-ALD mouse model. ${ }^{19}$ We used double mutants of $A b c d 1$ and its closest homolog, the Abcd2 gene $\left(A b c d 1^{-} / A b c d 2^{-1-}\right)$, as they present with a more severe AMN-like phenotype with earlier axonal degeneration at approximately 12 months of age. This model has been successfully used to assay pharmacological and gene therapeutic strategies to combat AMN. ${ }^{21,24,36}$ We generated

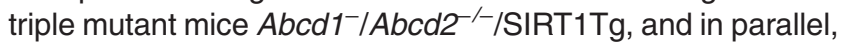
SIRT1 was pharmacologically activated by feeding $\mathrm{Abcd1}^{-}$/ $A b c d 2^{-1-}$ mice with RSV (Abcd1-/Abcd2 $\left.{ }^{-1-}+\mathrm{RSV}\right)$. Animals were then challenged with two behavioral tests. In the treadmill test, when $A b c d 1^{-} / A b c d 2^{-}$mice ran under conditions of high speed and high slope, the total number and duration of shocks were higher when compared with WT mice. Remarkably, animals with SIRT1 activation performed the test identical to control mice (Figures $5 \mathrm{a}$ and c). In the bar cross experiment, whereas double-knockout mice slipped off the bar more frequently and needed a longer time to reach the opposite platform, both $A b c d 1^{-} / A b c d 2^{-/} /$SIRT1Tg and Abcd $1^{-} / A b c d 2^{-/}+$RSV mice performed the task similarly to the control WT littermates (Figures $5 b, d$ ).

The activation of SIRT1 normalizes axonal degeneration in Abcd1-/Abcd2 ${ }^{-/}$mice. Finally, we evaluated the correlation between the observed improvements in the locomotor disability with arrested axonal degeneration, as assessed by immunohistochemical techniques. $A b c d 1^{-} / A b c d 2^{-/-}$mice presented an overt neuropathological phenotype characterized by the following: (i) microgliosis (Figures $6 \mathrm{a}$ and $\mathrm{b}$ ) and astrocytosis (Figures $6 \mathrm{e}$ and f), detected by Iba1 and GFAP staining, respectively; (ii) axonal damage suggested by the accumulation of synaptophysin (Figures $6 \mathrm{i}$ and $\mathrm{j}$ ) and amyloid precursor protein (APP) (Figures $6 \mathrm{~m}$ and $\mathrm{n}$ ) in axonal 
a

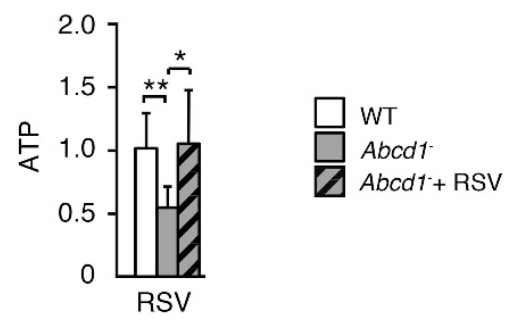

c

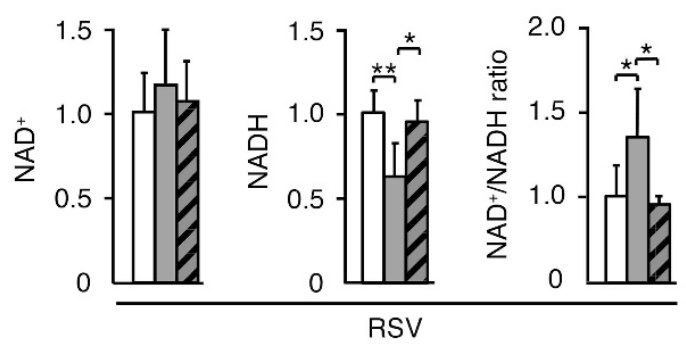

b

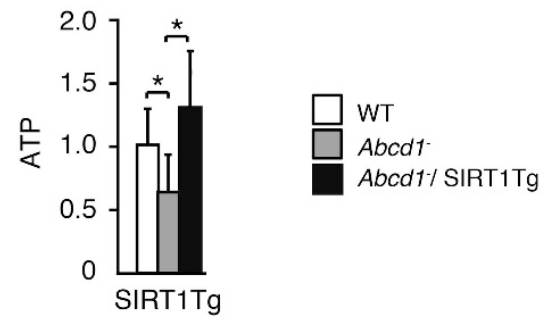

d
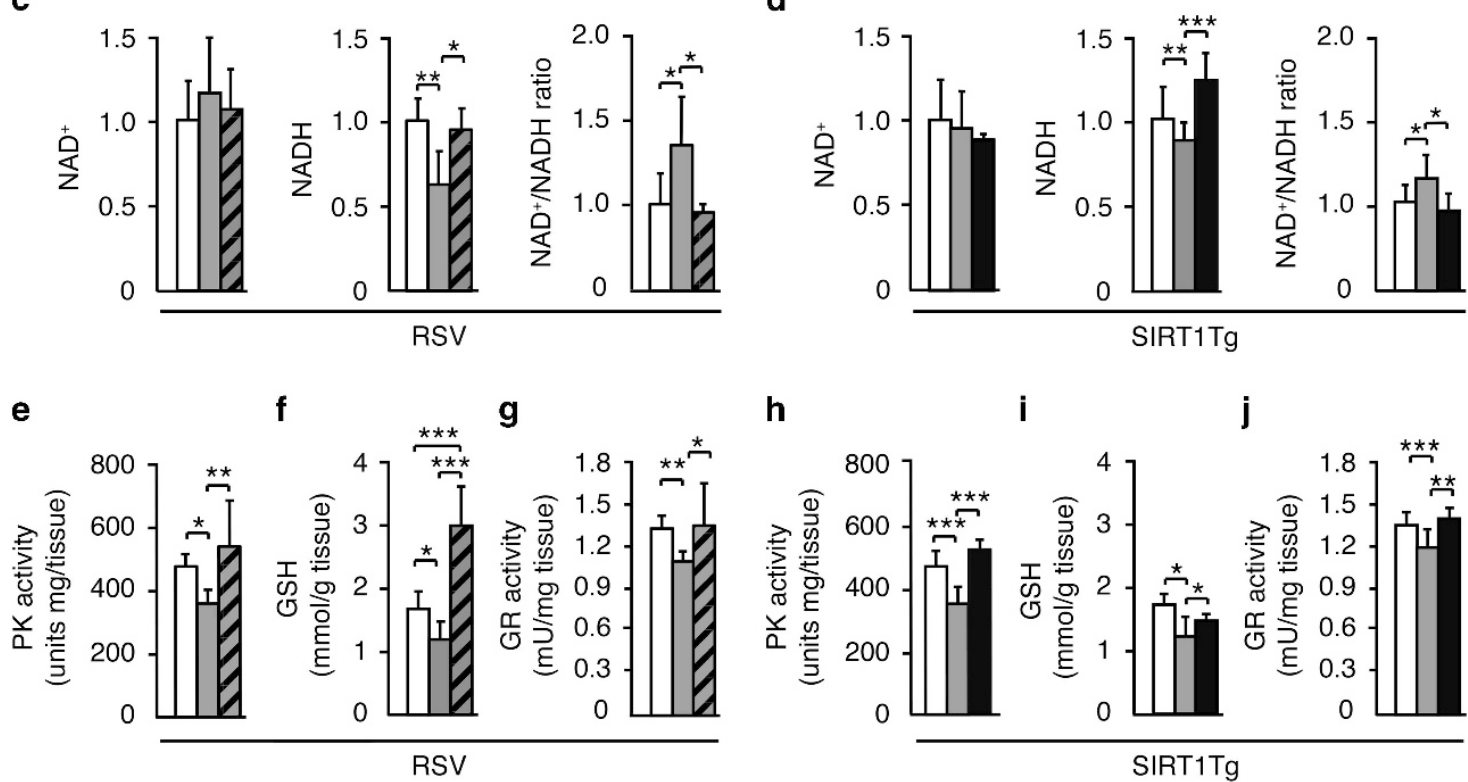

h $\quad$ i $\quad$ j

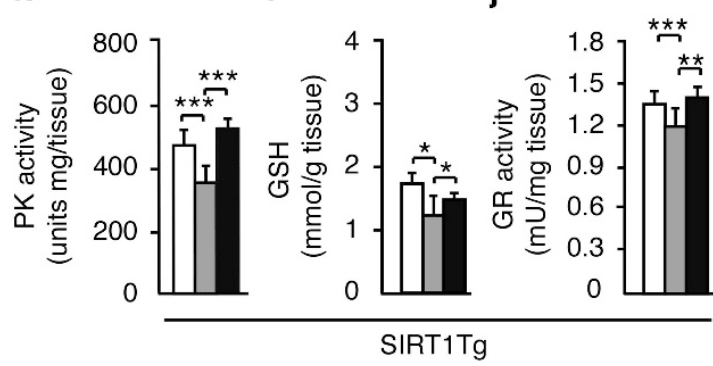

k

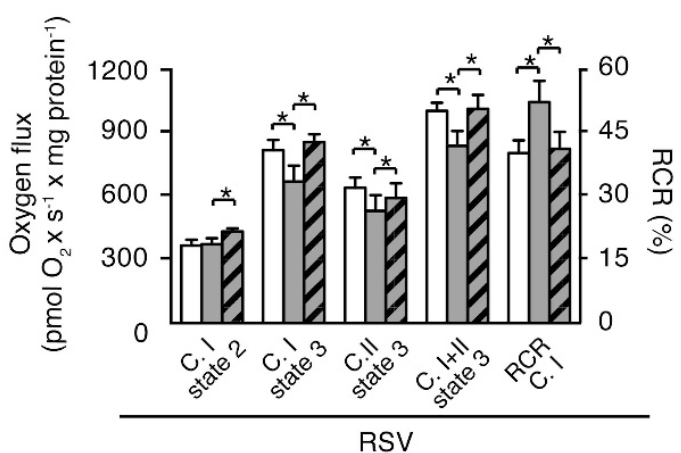

I

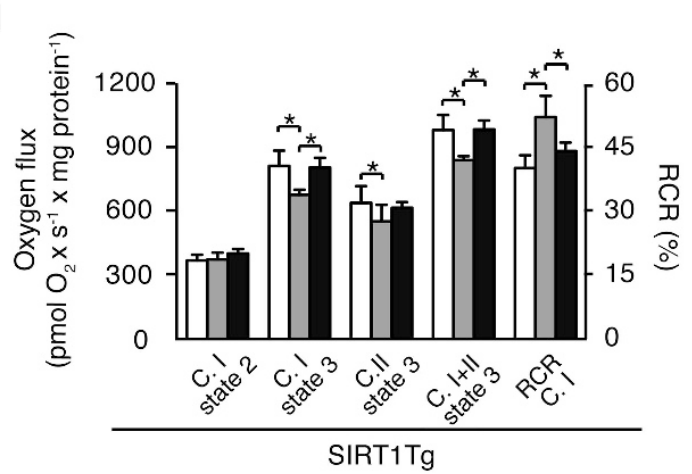

Figure 4 Activation of SIRT1 prevents energetic failure, promotes mitochondrial respiration and increases antioxidant defense in $A b c d 1^{-}$mice. (a-n) Energetic failure, antioxidant defense and mitochondrial respiration were measured in the spinal cord of 12-month-old (a, $\mathbf{c}, \mathbf{e}, \mathbf{f}, \mathbf{g}$ and $\mathbf{k})$ WT $(n=5), A b c d 1^{-}(n=6)$ and $A b c d 1^{-}$mice treated with RSV (Abcd1 $\left.{ }^{-}+\mathrm{RSV}\right)(n=6)$ and in 12-month-old (b, d, h, I, j and I) WT $(n=5), A b c d 1^{-}(n=6)$ and $A b c d 1^{-}$mice with transgenic overexpression of SIRT1 (Abcd1 $\left.{ }^{-} / \mathrm{SIRT} 1 \mathrm{Tg}\right)$ $(n=6)$. (a, b) ATP levels. Data are represented as fold change respect to WT mice. (c, d) NAD ${ }^{+}, N A D H$ and NAD $/ N A D H$ ratio. Data are represented as fold change with respect to WT mice. (e, h) PK activity. PK activity is represented as fold change with respect to WT mice. (f, i) GSH content. (g, j) GR activity. (k, I) Ex vivo mitochondrial respiration analysis performed on permeabilized sections of spinal cord. (C.I = complex I. C.II = complex II). Values are expressed as mean \pm S.D. $\left({ }^{*} P<0.05,{ }^{\star \star} P<0.01\right.$ and ${ }^{\star \star \star} P<0.001$, one-way ANOVA followed by Tukey's HSD post hoc)

swellings; (iii) scattered myelin debris detected with Sudan black (Figures $6 \mathrm{q}$ and $\mathrm{r}$ ); (iv) reduced SMl-32 staining as a marker of healthy motor neurons (Figures $6 \mathrm{u}$ and $\mathrm{v}$ ); (v) diminished amounts of cytochrome c (Figures 6y and $z$ ) that were used as an indicator of mitochondrial content; ${ }^{24}$ and (vi) increased staining of malondialdehyde (MDA), a lipoxidation marker (Figures 6c' and $\mathrm{d}^{\prime}$ ). We found that genetic or pharmacological SIRT1 activation in $A b c d 1^{-} / A b c d 2^{-/}$mice efficiently suppressed microgliosis (Figures 6a-d) and astrocytosis (Figures 6e-h). Moreover, synaptophysin (Figures 6i-I and $g^{\prime}$ ), APP accumulation (Figures $6 m-p$ and $g^{\prime}$ ) and myelin debris were prevented (Figures $6 q-t)$, indicating that SIRT1 activation halts axonal degeneration in the X-ALD mice. Moreover, these animals presented healthier motor neurons (Figures $6 u-x$ ), normalized mitochondrial content (Figures $\left.6 y-b^{\prime}\right)$ and reduced signs of lipooxidation (Figures $\left.6 c^{\prime}-f^{\prime}\right)$, 
a

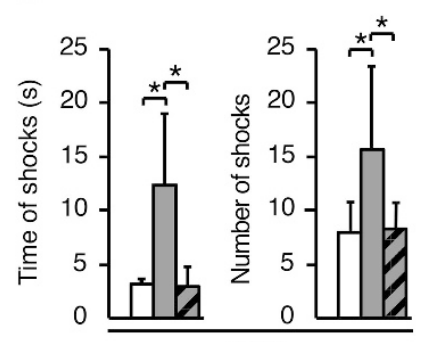

RSV

c

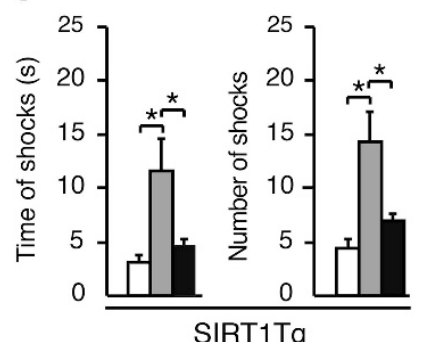

b

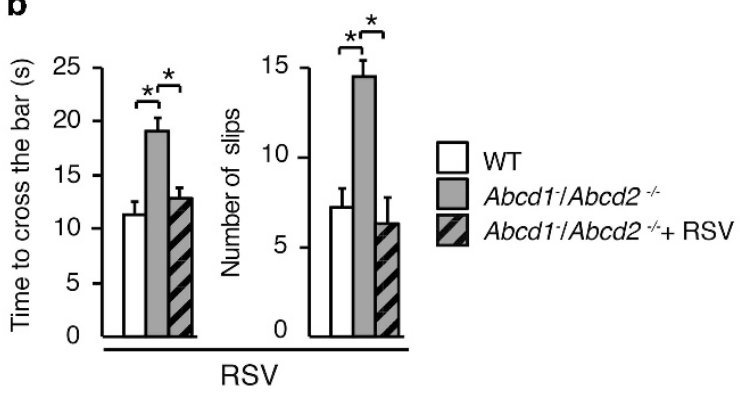

d

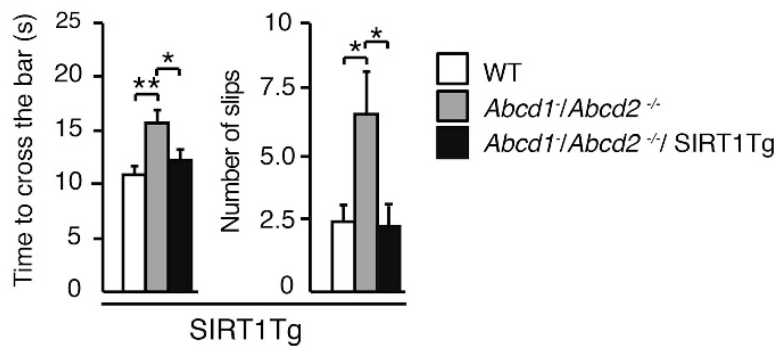

Figure 5 Activation of SIRT1 normalizes locomotor deficits in $A b c d 1^{-} / A b c d 2^{-/-}$mice. (a and c) Treadmill test was carried out in (a) 18-month-old WT ( $\left.n=12\right)$, $A b c d 1^{-} /$

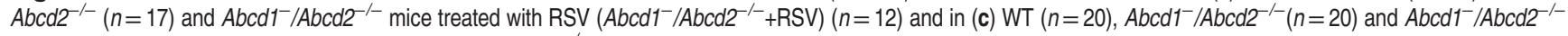
mice with transgenic overexpression of SIRT1 ( $A b c d 1^{-} / A b c d 2^{-}-$SIRT1Tg) $(n=16)$. The latency to falling off the belt (time of shocks) and the number of shocks received were computed after 7 min. (b and d) Bar-cross test was carried out in (b) 18-month-old WT ( $n=12), A b c d 1^{-} / A b c d 2^{-/-}(n=17)$ and $A b c d 1^{-} / A b c d 2^{-/-}$mice treated with RSV

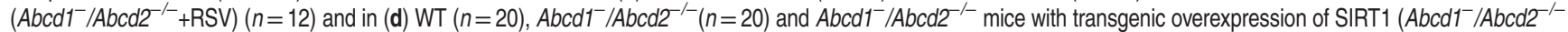
SIRT1Tg) $(n=16)$. The time spent to cross the bar and the numbers of slips were quantified. Values are expressed as mean \pm S.D. $\left({ }^{*} P<0.05,{ }^{* \star} P<0.01\right.$ and ${ }^{* \star \star} P<0.001$, one-way ANOVA followed by Tukey's HSD post hoc)

confirming the role of SIRT1 in preserving redox and metabolic homeostasis, and thus, axonal health.

\section{Discussion}

Oxidative stress and mitochondrial failure are intertwined, primary contributing factors to neurodegeneration and aging. In X-ALD, we have recently shown that the mitochondrial respiratory chain is the primary source of ROS, at least in human fibroblasts, and that impaired mitochondrial respiration and biogenesis underlies axonal degeneration in the spinal cord. It is tempting to speculate that a first hit (excessive mitochondrial ROS) may impair mitochondrial respiration and function, which in its turn may exacerbate ROS generation and mitochondrial failure in a vicious cycle. The inactivation of SIRT1 may come as an adaptive, finetuned response, reducing the generation of new mitochondria. Maintaining mitochondria numbers low may be advantageous for cellular homeostasis under conditions of acute stress such as ischemia/reperfusion, injury or when mitochondria are intrinsically impaired such as in mitochondrial-inherited genetic defects. In contrast, in the context of aging or neurodegenerative disorders with underlying chronic oxidative stress and progressive mitochondrial impairment, the situation may be different. Keeping SIRT1 active in order to generate new, intact mitochondria may be part of the solution, together with enhancing clearance of damaged mitochondria by mitophagy ${ }^{37}$ or by the proteasome. ${ }^{38}$

A large body of evidence suggests a central role of mitochondrial dysfunction associated with oxidative stress as culprits in the pathophysiology of chronic neurodegenerative disorders such as Alzheimer's disease (AD), Parkinson's disease (PD) or Huntington's disease (HD), ${ }^{39}$ in which SIRT1 may play a primary role. ${ }^{1}$ Thereby, a decrease in SIRT1 expression has been detected in $A D,{ }^{40} \mathrm{PD}^{41}$ and $H D^{42}$ patient brain samples, which is correlated with decreased PGC-1a activity. ${ }^{43-45}$ Indeed, the promotion of SIRT1 activity either genetically or pharmacologically with RSV, has been shown to combat several pathological hallmarks of neurodegeneration in a variety of models for $A D,{ }^{46-49} \mathrm{PD}^{50}$ and $H D,{ }^{51,52}$ supporting the neuroprotective notion of SIRT1 activation. However, recent studies have shown diametrically opposed results, indicating that selective SIRT1 inhibitors offer neuroprotection in cells and animal models of $\mathrm{HD},{ }^{8}$ or that overexpression of PGC-1a exacerbates $\beta$-amyloid and tau deposition in an AD mouse model. ${ }^{7}$ These latter examples illustrate the complexity of the scenario and preclude straightforward generalization and extrapolation of conclusions from one model to another from arising, while inviting to a cautious examination of the specific pathomechanisms operating in every particular disease model.

Although chronic and slowly progressive axonal degeneration is a prominent contributor to disability in neurodegenerative diseases, ${ }^{9,53}$ the impact of SIRT1 activation in axonal mitochondria or in dying back axonal degeneration still remains unexplored. In axons, mitochondria provide a substantial part of the energy demanded during the neurotransmission. Nonetheless, axonal mitochondria are more vulnerable than their cellular counterparts. First, mitochondria require from an elongated shape to fit with the axon morphology. Second, axonal mitochondria are discrete organelles rather than reticular-shaped structures and thus, 
more reliant on fusion-fission events to exchange material among them. Third, the transport of the mitochondria through the long axonal compartment towards ATP-demanding areas has to be tightly regulated.

An imbalance in the triad ROS/ATP/calcium that overcomes the homeostatic capacity of the mitochondria initiates a toxic cycle of events that will ultimately activate the axonal degeneration program. For instance, mitochondrial ROS may oxidize and inhibit the OXPHOS system, inducing a decline in the ATP production. This energetic failure, in its turn, impairs the ATP-dependent ionic pumps, triggering a massive calcium influx. Furthermore, ROS not only impair the OXPHOS system but also promote the opening of the mtPTP leading to a subsequent efflux of calcium from the mitochondria to the cytosol. Cytoplasmic calcium overload causes activation of calcium-dependent calpains. These proteases disassemble the microtubule structure and impair the correct transport along the axon. All these events will result in axonal swelling, loss of axonal continuity and eventually, axonal destruction. $^{9}$ This susceptibility of axonal mitochondria is clearly exemplified by primary mitochondrial diseases such as the peripheral neuropathy Charcot-Marie-Tooth or in several Hereditary Spastic Paraplegias (HSP). In the latter entities, axonal degeneration of the corticospinal tracts is the predominant neuropathological feature, $, 54,55$ thus closely resembling the clinical phenotype of X-AMN patients.

In conclusion, mitochondria are critical players for the maintenance of axonal integrity. In this sense, activation of pathways such as SIRT1/PGC-1a that maintain a healthy mitochondrial population (that is, activating redox-stress responses, boosting mitochondrial respiration, increasing ATP production and regulating mitophagy) arises as a powerful strategy to halt axonal degeneration.

The first evidences of the protective role of SIRT1 on axonal integrity come from studies on the Wallerian degeneration slow (Wlds) mice. The chimeric protein Wlds fuses a $\mathrm{N}$-terminal sequence of the multiubiquitination factor Ube $4 \mathrm{~b}$ with the $\mathrm{NAD}^{+}$-synthesizing enzyme nicotinamide mononucleotide adenylyl transferase1 (Nmnat1). ${ }^{56}$ The first studies attributed the axonal protection observed in Wids mice, to an increased activity of Nmnat1, and a subsequent increment in the biosynthesis of $\mathrm{NAD}^{+}$. Inhibition of SIRT1 in an in vitro model of Wallerian degeneration showed no protection to axonal degeneration and thus authors claimed that axonal protection was mediated by SIRT1. However, later studies cast doubts on the former conclusions, as they showed that RSV and SIRT1 were not directly involved in these processes. Indeed, RSV abolished resistance to axonal degeneration, ${ }^{57}$ and $\mathrm{NAD}^{+}$or Wlds were able to protect axonal degeneration in $S I R T 1^{-/}$cultures, most likely because $\mathrm{NAD}^{+}$and WIds delayed the decrease of the ATP level in transected axons. ${ }^{58}$ Furthermore, axonal degeneration was prevented by $\mathrm{NAD}^{+}$ via mitochondrial SIRT3 rather than SIRT1. ${ }^{59}$ It is worth to mention that mechanisms of neuronal death and axonal degeneration often differ. For instance, the Wlds protein has no effect on survival of the neuronal soma. ${ }^{60}$ These results stress the importance of understanding which specific mechanisms underlie axonal degeneration and axonal protection. To our knowledge, our work is the first to provide in vivo evidence of SIRT1 activation preventing axonal degeneration in a mouse model of non-immune-mediated axonopathy.

On the other hand, the results of the present study concur with previous reports showing that, in contrast to the prooxidant effects exerted by high levels of SIRT1 overexpression (approximately 12-fold), a more moderate expression of SIRT1 is protective against oxidative stress by inducing antioxidant defenses via FOXO1 deacetylation in the heart muscle. ${ }^{61}$ In the same line, RSV has been reported to normalize GSH or MDA levels in a rat model of spinal cord injury ${ }^{62}$ or in the brain of obese mice. ${ }^{63}$

Since the discovery of sirtuin-activating compounds such as $\mathrm{RSV}^{4}$ a rich controversy exists regarding the direct path via SIRT1 or via an indirect modus operandi of these compounds. ${ }^{34} \mathrm{~A}$ recent and elegant study has shed some light on the debate and demonstrated that SIRT1 is directly activated by RSV, through an allosteric mechanism. ${ }^{64}$ However, this effect may be dependent on the cell type, the dose of RSV used or other factors related to the specific context. In our experimental in vitro conditions, RSV inhibited ROS production independently of SIRT1, which may be attributed to the polyphenolic nature of the compound, exhibiting well-known ROS scavenger properties. In contrast, other effects such as prevention of cell death in galactose, prevention of mitochondria depolarization and recovery of the ATP levels were mediated by SIRT1. In the preclinical test in the X-ALD mouse, the results suggest that RSV prevents oxidative lesions, mitochondrial dysfunction and axonal degeneration in the same way as the overexpression of SIRT1, most likely in a SIRT1-dependent manner. In conclusion, our results indicate that the twofold overexpression of SIRT1 is sufficient to restore metabolic and redox homeostasis and to prevent axonal degeneration in the X-ALD mouse model.

Collectively, our data strongly suggest that an early and carefully tailored intervention using RSV (or other SIRT1 activators) may be a plausible therapeutic option for X-AMN patients who do not suffer from severe neuroinflammatory demyelination. Biological effects are easily monitored by quantitative measurement of oxidative damage biomarkers in plasma $^{29}$ or red blood cells. ${ }^{30} \mathrm{RSV}$ has been proven to improve the overall metabolic health status in clinical trials for patients with metabolic disorders, ${ }^{32}$ which may or may not be confirmed in currently ongoing clinical trials for AD and Friedreich ataxia (NCT01339884, NCT01504854, NCT00743743, NCT00678431). Therapeutic implications derived from this work may be potentially extended to other diseases such as multiple sclerosis, ${ }^{65}$ amyotrophic lateral sclerosis $^{66}$ and some forms of HSP such as SPG7, ${ }^{54}$ which share axonal degeneration as a primary component of clinical disability and oxidative stress/mitochondria impairment as the primary or early contributing pathogenic factors.

\section{Materials and Methods}

Human brain samples. Brain tissues (unaffected white matter of frontal lobes from patients and controls, plus affected white matter of parietal and occipital lobes from cerebral AMN patients) were obtained from the Brain and Tissue Bank for Developmental Disorders at the University of Maryland, Baltimore, MD, USA. ${ }^{26}$ The frontal cortex from control subjects was obtained from the Institute of Neuropathology Brain Bank (HUB-ICO-IDIBELL Biobank) following the guidelines of both Spanish legislation and the local ethics committee. The postmortem interval 

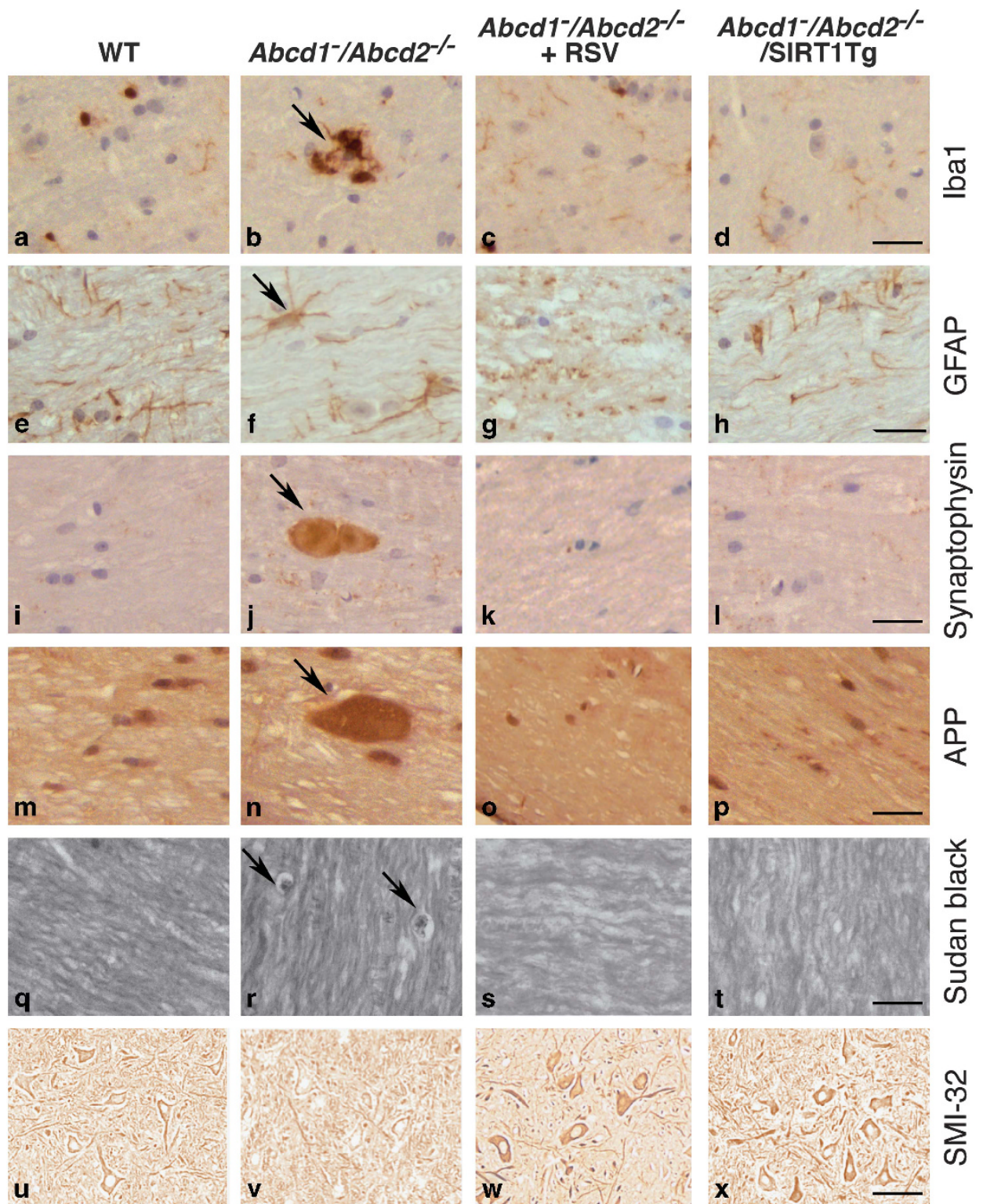

$\frac{0}{\stackrel{4}{1}}$
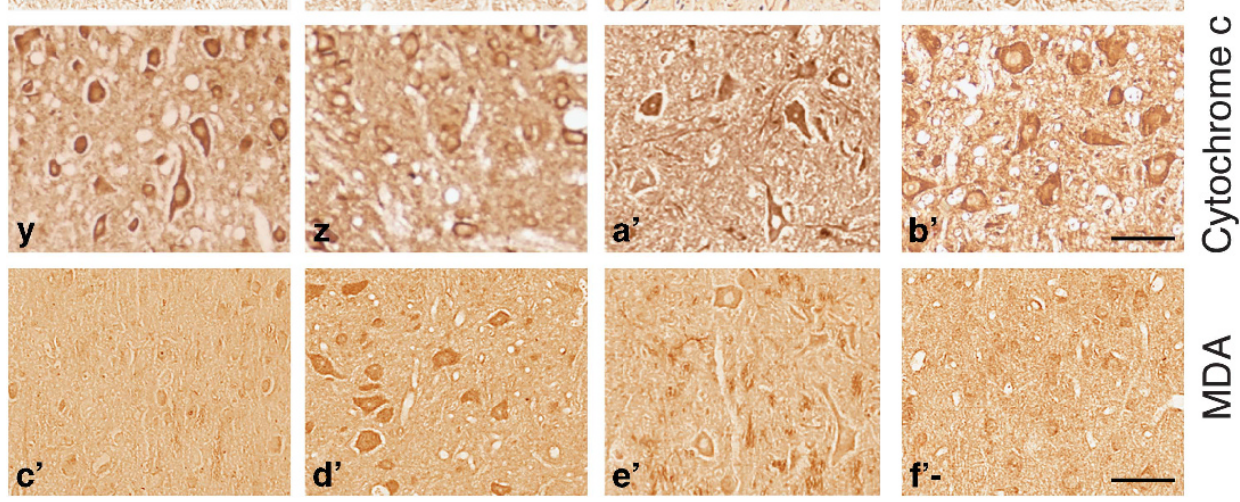

Figure 6 Activation of SIRT1 normalizes axonal degeneration in $A b c d 1^{-} / A b c d 2^{-/-}$mice. (a-f') Immunohistological analysis of axonal pathologies performed in 18-month-old

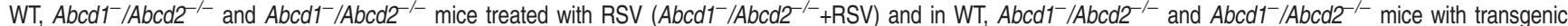

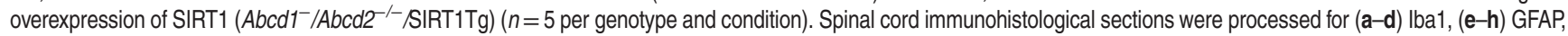

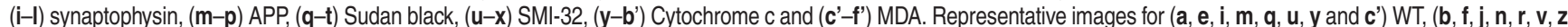
and $\mathbf{d}^{\prime}$ ) for $A b c d 1^{-} / A b c d 2^{-/-},\left(\mathbf{c}, \mathbf{g}, \mathbf{k}, \mathbf{0}, \mathbf{s}, \mathbf{w}, \mathbf{a}^{\prime}\right.$ and $\left.\mathbf{e}^{\prime}\right)$ for $A b c d 1^{-} / A b c d 2^{-1-}+\mathrm{RSV}$ and $\left(\mathbf{d}, \mathbf{h}, \mathbf{l}, \mathbf{p}, \mathbf{t}, \mathbf{x}, \mathbf{b}\right.$ ' and $\left.\mathbf{f}^{\prime}\right)$ for $A b c d 1^{-} / A b c d 2^{-/} / S I R T 1 T g$ mice are shown. Scale bar $=25 \mu \mathrm{m}$. (g') Quantification of synaptophysin and APP accumulation in spinal cord immunohistological sections of WT, $A b c d 1^{-} / \mathrm{Abcd2}^{-/}, \mathrm{Abcd1}^{-} / \mathrm{Abcd2^{-/ }}+\mathrm{RSV}^{-}$and Abcd1 $1^{-} / A b c d 2^{--} /$SIRT1Tg mice. Values are expressed as mean \pm S.D. $\left({ }^{\star} P<0.05,{ }^{* \star} P<0.01\right.$ and ${ }^{\star \star \star} P<0.001$, one-way ANOVA followed by Tukey's HSD post hoc) 


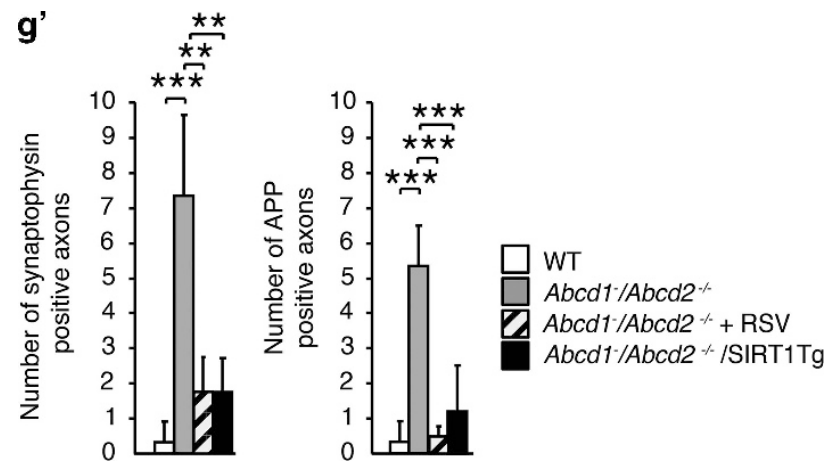

Figure 6 Continued

between death and tissue processing was between 6 and $12 \mathrm{~h}$ in all cases. Informed written consent was obtained from all patients or their legal representatives, and the local ethics committee approved the studies.

Mouse strains. Two X-ALD mouse models were used in this study. The first model was $A b c d T^{-}$mice at 12 months of age. These mice already show biochemical signs of pathology, including oxidative stress ${ }^{20}$ and alterations in energy homeostasis, ${ }^{23}$ although the first clinical signs of AMN (that is, axonopathy and locomotor impairment) appear at 20 months. ${ }^{18,19}$ We characterized the biochemical signs of adult X-ALD in these mice. The second model was mice having a double gene knockout of both the Abcd1 and $\mathrm{Abcd2}$ transporters $\left(\mathrm{Abcd1^{- }} / \mathrm{Abcd2^{-1 }}\right)$. The $\mathrm{Abcd1}^{-} / \mathrm{Abcd2}^{-/}$mice, compared with the $A b c d t^{\top}$ mice, display greater VLCFA accumulation in the spinal cord ${ }^{19}$ higher levels of oxidative damage to proteins ${ }^{20}$ and a more severe AMN-like pathology with an earlier onset at 12 months of age. ${ }^{19,67}$ It is worth mentioning that to date, no disease-causative role for $A B C D 2$ has been found, although its absence provokes a partially overlapping fatty acid pattern when compared with the ABCD1-dependent biochemical phenotype. ${ }^{68,69}$ We assessed the clinical signs of AMN in these double $\mathrm{Abcd1}^{-}$/ $A b c d 2^{--}$mice. The mice used for the study were of a pure C57BL/ $6 \mathrm{~J}$ background. SIRT1 transgenic mice (SIRT1Tg mice) were a gift from Manuel Serrano. ${ }^{5}$ The animals were killed, and the tissues were recovered and stored at $-80^{\circ} \mathrm{C}$. All of the methods employed in this study were in accordance with the Guide for the Care and Use of Laboratory Animals published by the U.S. National Institutes of Health (NIH Publications No. 85-23, revised 1996) and the guidelines of the ethical committees of IDIBELL and the Generalitat de Catalunya, Spain.

Treatment of the mice. Resveratrol (RSV) (Orchid Chemicals \& Pharmaceuticals Ltd, Chennai, India) $(0.04 \%$ w/w) was mixed into AIN-93G chow from Dyets (Bethlehem, PA, USA) to provide a dose of $400 \mathrm{mg} / \mathrm{kg} /$ day. ${ }^{6}$ For the characterization of biochemical signs of adult X-ALD, 10 and a half-month-old animals were randomly assigned to one of the following dietary groups for 7 weeks. Group l: wild-type (WT) mice received normal AIN-93G chow $(n=8)$; group II: $A b c d 1^{-}$mice received normal AIN-93G chow $(n=8)$; group III: $A b c d 1^{-}$mice were treated with chow containing RSV $(n=8)$. For clinical signs of AMN, 12-month-old animals were randomly assigned to one of the following dietary groups for 6 months. Group I: WT mice received normal AIN-93G chow ( $n=12)$; group II: $A b c d 1^{-} \mid A b c d 2^{-1}$ mice received normal AIN-93G chow ( $n=17)$; group III: $A b c d 1^{-} / A b c d 2^{-/-}$mice were treated with chow containing RSV $(n=12)$. RSV had no effect on weight or food intake under any treatment protocol.

SIRT1 activity. SIRT1 activity was measured using the fluorometric assay kit (BIOMOL, Enzo Life Sciences International, Inc., Farmingdale, NY, USA) based on the generation of a fluorophore via the NAD+-dependent deacetylation by SIRT1 of its substrate. The fluorescence signal generated by the assay is proportional to the Lys382 deacetylation. In brief, $25 \mu \mathrm{g}$ total protein was incubated with Fluor de Lys-Sirt1 substrate $(100 \mu \mathrm{M})$ and $\operatorname{NAD}^{+}(100 \mu \mathrm{M})$ at $37^{\circ} \mathrm{C}$ for $30 \mathrm{~min}$ in a final volume of $50 \mu \mathrm{l}$. The reaction was stopped by the addition of $50 \mu \mathrm{l}$ developer reagent and $2 \mathrm{mM}$ NAM, and the fluorescence was subsequently monitored for $30 \mathrm{~min}$ at wavelengths of 360 $\mathrm{nm}$ (excitation) and $460 \mathrm{~nm}$ (emission). ${ }^{70}$

Quantitative real-time PCR. The total RNA was extracted using the RNeasy Kit (Qiagen, Hilden, Germany). The total DNA was extracted using the Gentra Puragene Tissue Kit (Qiagen). Gene expression and mitochondrial DNA (mtDNA) and nuclear DNA (nDNA) levels were measured by TaqMan quantitative real-time PCR (RT-PCR). To quantify mRNA levels, $1 \mu \mathrm{g}$ of RNA was transcribed into cDNA using Superscript II reverse transcription reagents in a final volume of $25 \mu \mathrm{l}$ (Invitrogen, Thermo Fisher Scientific Inc., Waltham, MA, USA). 0.1-0.2 $\mu$ l of cDNA or $100 \mathrm{ng}$ of DNA were used to measure mRNA or mtDNA levels, respectively. Standardized primers used for RT-PCR were: mouse SIRT1 (Mm00490758), mouse PGC-1 $\alpha$ (Mm00447183), mouse NRF-1 (Mm00447996) and mouse TFAM (Mm00447485). Expression of the genes of interest was normalized to that of the reference control mouse RPLO (Mm01974474). To quantify mouse mtDNA content, primer for mouse cytochrome b (cytb) were designed (Custom TaqMan Gene Expression Assays; Applied Biosystems, Thermo Fisher Scientific Inc.). The sequences for mouse cytb were: ATGACCCCAATACGCAAAATTA (forward) and GGAGGACATAGCCTATGAAGG (reverse) and the FAM-labeled probe was TTGCAACTATAGCAACAG. Quantification of mtDNA was referred to nDNA as determined by the amplification of the intronless mouse nuclear gene C/EBP $\alpha$ (Mm00514283). ${ }^{24}$ Each sample was run in duplicate, and the mean value of the duplicate was used to calculate the mtDNA amount or mRNA expression using the comparative $\left(2^{-\Delta C t}\right)$ method, according to the manufacturer's instructions.

Immunoblot. The tissues were removed from the killed mice and flash-frozen in liquid nitrogen. The frozen tissues were homogenized in RIPA buffer, boiled for 5 min and centrifuged. Ten to $100 \mu \mathrm{g}$ were loaded on to each lane of $10 \%$ polyacrylamide gels for $60 \mathrm{~min}$ at $120 \mathrm{mV}$. Resolved proteins were transferred onto nitrocellulose membrane. Proteins were detected with ECL western blotting analysis system (GE Healthcare, Buckinghamshire, UK) followed by exposure to CL-XPosure Film (Thermo Fisher Scientific Inc.) as earlier described. ${ }^{23}$ Primary antibodies used for immunoblot were: rabbit anti-SIRT1: dilution 1:5000 (ab32441 (Abcam plc, Cambridge, UK)); mouse anti-NADH-ubiquinol oxidoreductase (NDUFB8 (complex I)): dilution 1/2000 (459210 (Molecular Probes, Thermo Fisher Scientific Inc.)); mouse anti-succinate dehydrogenase (ubiquinone) iron-sulfur subunit (SDHB (complex II)): dilution 1/2000 (459230 (Molecular Probes); mouse anti-Porin 31HL (VDAC or Voltage-Dependant Anion Channel): dilution 1/1000 (529536 (Merck Millipore, Merck KGaA, Darmstadt, Germany)); rabbit anti-pyruvate kynase: dilution 1/500 (ab38237 (Abcam plc)). Goat anti-rabbit IgG linked to horseradish peroxidase, dilution: 1/15000 (P0448 (Dako, Glostrup, Denmark)) and goat anti-mouse IgG linked to horseradish peroxidase, dilution: 1/15000 (G21040 (Invitrogen)) were used as secondary antibodies.

Metabolic failure determination. The GSH levels were determined by $\mathrm{Q}-\mathrm{TOF}$ analysis. NAD+/NADH was measured by the NAD ${ }^{+}$cycling assay. The ATP levels were measured by a chemiluminescence system using ATPlite 1step (PerkinEImer, Inc., Waltham, MA, USA). ${ }^{23}$ GR and PK activity were determined with a spectrophotometrical method. ${ }^{24}$

High-resolution respirometry. Animals were killed by cervical dislocation after being fasted overnight. Spinal cords were rapidly excised, rinsed with ice-cold normal saline and cut into slices with a tissue chopper adjusted to cut a width of $350 \mu \mathrm{m}$. About 12-15 slices of the lumbar region were collected and transferred quickly into individual wells of a 6-well tissue culture plate containing $2 \mathrm{ml}$ of ice-cold permeabilization medium (in $\mathrm{mM}: 7.23$ potassium ethylene glycol-bis (b-aminoethyl ether) $N, N, N^{\prime}, N^{\prime}$-tetraacetate (K $\mathrm{K}_{2}$ EGTA), $2.77 \mathrm{CaK}_{2}$ EGTA, 60N,N-bis[2hydroxyethyl]- 2-aminoethanesulfonic acid (BES), $5.69 \mathrm{MgATP}, 20$ taurine, $3 \mathrm{~K}_{2} \mathrm{HPO}$, 
0.5 dithiothreitol and 81 potassium methanesulfonate, $\mathrm{pH} 7.1$ at $25^{\circ} \mathrm{C}$ ), rinsed and immediately transferred again into another well with the same medium containing $20 \mu \mathrm{l}$ of saponin stock solution $(5 \mathrm{mg} / \mathrm{ml}$; final concentration $50 \mu \mathrm{g} / \mathrm{ml})$. Lumbar spinal cord slices were shaken by gentle agitation in the cold room (on ice) for $30 \mathrm{~min}$. Afterwards, all samples were quickly transferred from the saponin permeabilization medium into $2 \mathrm{ml}$ of respiration medium (in mM: 7.23 $\mathrm{K}_{2}$ EGTA, 2.77 CaK $\mathrm{K}_{2} \mathrm{EGTA}, 100$ potassium salt of 2-(N-morpholino)ethanesulfonic acid (K-MES), $1.38 \mathrm{MgCl}_{2}, 20$ taurine, $3 \mathrm{~K}_{2} \mathrm{HPO} 4,0.5$ dithiothreitol, 20 imidazole and $5 \mathrm{mg} / \mathrm{ml}$ bovine serum albumine (BSA), pH 7.1 at $25^{\circ} \mathrm{C}$ ), and shaken by gentle agitation for $10 \mathrm{~min}$ in the cold room (on ice) before performing respirometry. The oxygen flux of permeabilized lumbar spinal cord slices ( $n=4-6$ per genotype) was measured at $37^{\circ} \mathrm{C}$ by highresolution respirometry using an Oxygraph-2k (Oroboros Instruments, Innsbruck, Austria) with chamber volumes set at $2 \mathrm{ml}$. In order to avoid tissue disaggregation, lumbar spinal cord slices required setting a slow bar stirring speed (150 r.p.m.), but not too low to compromise homogeneity of substrate and oxygen concentrations in the measuring chambers, and therefore signal stability. DatLab software (Oroboros Instruments) was used for data acquisition (2s time intervals) and analysis, which includes calculation of the time derivative of oxygen concentration and correction for instrumental background oxygen flux. ${ }^{25}$ Initially, we measured endogenous respiration in the absence of additional substrates. For the evaluation of the relative contributions of mitochondrial complexes to oxygen consumption, several specific mitochondrial inhibitors and substrates were added sequentially as routinely performed in our laboratory ${ }^{25}$ and calculated as steady-state respiratory flux in the time interval between 5 and $10 \mathrm{~min}$ after its addition. First, we added glutamate $(10 \mathrm{mM})$ and malate $(5 \mathrm{mM})$ to increase NADH levels in order to measure the complex I nonphosphorylative activity, or state 2. ADP $(10 \mathrm{mM})$ was added to quantify the complex I-dependent phosphorylative activity, or state 3. Immediately afterwards, we added succinate $(10 \mathrm{mM})$, which is the substrate for complex II. At this point, the level of oxygen consumption corresponded to complex I- and II-dependent phosphorylative activity. The addition of $0.5 \mu \mathrm{M}$ rotenone inhibits complex I; therefore, oxygen consumption measured after the addition of rotenone only reflects complex IIdependent phosphorylative activity (in absence of electron back flux to complex I). Finally, complex III activity was inhibited with antimycin A $(2.5 \mu \mathrm{M})$. In order to avoid oxygen limitations, all the experiments were performed above $50 \%$ oxygen saturation. Oxygen consumption was normalized by actual protein content in the respirometer chambers using the Bradford assay.

Analysis of locomotion. Locomotor deficits were evaluated with the treadmill test and the bar cross test.

Treadmill test. Treadmill apparatus (Panlab, Barcelona, Spain) consisted of a belt (50 cm long and $20 \mathrm{~cm}$ wide) varying in terms of speed $(5-150 \mathrm{~cm} / \mathrm{s}$ ) and slope $\left(0-25^{\circ}\right)$ enclosed in a plexiglass chamber. ${ }^{24}$ An electrified grid was located to the rear of the belt on which footshocks $(0.2 \mathrm{~mA})$ were administered whenever the mice fell off the belt. The mice were placed on the top of the already moving belt facing away from the electrified grid and in the direction opposite to the movement of the belt. Thus, to avoid the footshocks, the mice had to locomote forward. The latency to falling off the belt (time of shocks in s) and the number of received shocks were measured. The mice were evaluated in five trials in a single-day session. In the first trial, the belt speed was set at $20 \mathrm{~cm} / \mathrm{s}$ and the inclination at $5^{\circ}$. In the second and third trial, the belt speed was $10 \mathrm{~cm} / \mathrm{s}$ and the slope was increased to $10^{\circ}$ and $20^{\circ}$, respectively. Then, for the fourth and the fifth trials, the inclination was maintained at $20^{\circ}$ and the belt speed was increased to 20 and $30 \mathrm{~cm} / \mathrm{s}$, respectively. For the three first trials, mice ran for $1 \mathrm{~min}$. For the fourth and fifth tests, time of the experiment was 3 and $7 \mathrm{~min}$, respectively. The time between each test was 1, 1, 5 and $20 \mathrm{~min}$, respectively. When the mice were subjected to consecutive trials at increasing speeds of up to $20 \mathrm{~cm} / \mathrm{s}$ and a $20^{\circ}$ slope, no differences were detected from one session to another between the WT and $A b c d 1^{-} / A b c d 2^{-/}$mice. However, when the belt speed was increased up to $30 \mathrm{~cm} / \mathrm{s}$ and the slope was $20^{\circ}$, differences were detected between the $A b c 1^{-} / \mathrm{Abcd2}^{-/}$mice and the controls because this task requires greater coordination. These conditions were therefore chosen to assess the effects of resveratrol.

Horizontal bar cross test. The bar cross test was carried out using a wooden bar of $100 \mathrm{~cm}$ in length and $2 \mathrm{~cm}$ in width (diameter). This bar is just wide enough for mice to stand on with their hind feet hanging over the edge such that any slight lateral misstep will result in a slip. The bar was elevated $50 \mathrm{~cm}$ from the bench surface, so that animals did not jump off, yet were not injured upon falling from the bar. The mice are put on one end of the bar and expected to cross to the other end. To eliminate the novelty of the task as a source of slips, all animals were given four trials on the bar the day before and at the beginning of the testing session. In an experimental session, the numbers of hind limb lateral slips and the time to cross the bar were counted on four consecutive trials.

Immunohistochemistry. Spinal cords were harvested from 18-month-old WT, $A b c d 1^{-} / A b c d 2^{-/}, A b c d 1^{-} / A b c d 2^{-/} / S I R T 1 T g$ and WT, $A b c d 1^{-} / A b c d 2^{-/}$and $A b c d 1^{-} / A b c d 2^{-1}$ mice fed with RSV for 6 months after the perfusion with $4 \%$ PFA. Spinal cords were embedded in paraffin and serial sections ( $5 \mu \mathrm{m}$ thick) were cut in a transversal or longitudinal $(1 \mathrm{~cm}$ long) plane. The number of abnormal specific profiles was counted at every 10 sections for each stain. At least three sections of the spinal cord were analyzed per animal and per stain. The sections were stained with hematoxylin and eosin and Sudan black, or processed for immunohistochemistry to rabbit anti-lba1: dilution 1/1000 (019-19741 (Wako Pure Chemicals Industries, Ltd., Osaka, Japan)); rabbit anti-glial fibrillary acidic protein (GFAP): dilution 1/300 (Z-0334 (Dako)); mouse anti-synaptophysin: dilution 1/500 (SYNAP-299- L-CE (Leica Biosystems, Nussloch, Germany)); rabbit anti- amyloid precursor protein (APP): dilution 1/100 (AHP538 (AbD Serotec, Oxford, UK)); mouse anticytochrome c: dilution 1/100 (55643 (BD Biosciences Pharmingen, San Diego, CA, USA)); mouse anti-neurofilament $H$ non-phosphorylated (SMI32): dilution 1/3000 (SMI-32P (Covance Antibody, BioLegend, Dedham, MA, USA)); rabbit anti-MDA: dilution $1 / 1000 .{ }^{21,24,67}$ The mounting medium used for the acquisitions of the images was DPX. Images were acquired with Olympus BX51 microscope (UPlan FL $\mathrm{N} 20 \times / 0.50 \mathrm{Ph} 1$ ) (Olympus Corporation, Tokyo, Japan) connected to a Olympus DP71 camera and Cell/B software (Olympus Corporation).

Cell culture experiments. Human control and X-ALD fibroblasts were obtained after informed consent at the Bellvitge University Hospital. The cells were grown in DMEM containing $10 \%$ of fetal bovine serum, $100 \mathrm{U} / \mathrm{ml}$ penicillin and $100 \mathrm{mg} / \mathrm{ml}$ streptomycin and maintained at $37{ }^{\circ} \mathrm{C}$ in humidified $95 \%$ air $/ 5 \% \mathrm{CO}_{2}$. To assess the effect of the drugs, the cells were grown in DMEM medium (without glucose, pyruvate and L-glutamine) supplemented either with $1 \mathrm{~g} / \mathrm{l}$ of glucose or $1 \mathrm{~g} / \mathrm{l}$ of galactose. ${ }^{26}$ The compounds tested were added to cells at $80-90 \%$ confluence with the following concentrations: C26:0 (Sigma-Aldrich Corporation, St. Louis, MO, USA) $=50 \mu \mathrm{M}$; RSV (Sigma-Aldrich Corporation) $=25 \mu \mathrm{M}$ and the SIRT1 inhibitor EX-527 (Tocris Bioscience, Bristol, UK $)=10 \mu \mathrm{M}$. The evaluation of ROS, cell death, ATP and $\Delta \Psi \mathrm{m}$ quantification by flow cytometry were performed as previously described. ${ }^{26}$

Statistical analysis. The values are expressed as the mean \pm S.D. The significant differences were determined by a two-tailed unpaired Student's $t$ test or one-way ANOVA followed by Tukey's HSD post hoc $\left({ }^{*} P<0.05\right.$, ${ }^{* *} P<0.01$, $\left.{ }^{* \star *} P<0.001\right)$ after verifying normality. Statistical analyses were performed using the SPSS 12.0 software program.

\section{Conflict of Interest}

The authors declare no conflict of interest.

Acknowledgements. This work was supported by grants from the European Commission [FP7-241622], the Spanish Institute for Health Carlos III [FIS PI11/01043], the Oliver's Army Foundation, the Government of Catalonia [2009SGR85 to AP], the Spanish Institute for Health Carlos III [Miguel Servet program CP11/00080 to S.F.], the European Leukodystrophy Association (ELA) [ELA 2010-020F1 to SF, ELA 2013-003F1 to LM], the Spanish Ministry of Education [FPU program AP2008-03728 to LM]. JG held an IDIBELL PhD fellowship. The studies conducted at the Experimental Medicine Department were supported in part by R+D grants from the Spanish Ministry of Science and Innovation [BFU2009-11879/BFI], the Spanish Ministry of Health [PI081843, PI1300584], the Government of Catalonia [2009SGR735], the 'La Caixa' Foundation and COST B35 Action of the European Union. CIBERER and CIBERNED are initiatives of the Spanish Institute for Health Carlos III.

\section{Author contributions}

LM, SF and AP participated in the study design. LM, MR, JG, CG and SF carried out all experimental assays except histopathological studies, performed by NYC and FB. MJ and AN performed GSH quantification and JB performed high-resolution respirometry. LM and AP wrote the manuscript. All authors reviewed the manuscript.

1. Donmez G. The neurobiology of sirtuins and their role in neurodegeneration. Trends Pharmacol Sci 2012; 33: 494-501. 
2. Scarpulla RC, Vega RB, Kelly DP. Transcriptional integration of mitochondrial biogenesis. Trends Endocrinol Metab 2012; 23: 459-466.

3. Haigis MC, Sinclair DA. Mammalian sirtuins: biological insights and disease relevance. Annu Rev Pathol 2010; 5: 253-295.

4. Howitz KT, Bitterman KJ, Cohen HY, Lamming DW, Lavu S, Wood JG et al. Small molecule activators of sirtuins extend Saccharomyces cerevisiae lifespan. Nature 2003; 425: 191-196.

5. Herranz D, Munoz-Martin M, Canamero M, Mulero F, Martinez-Pastor B, Fernandez-Capetillo $\mathrm{O}$ et al. Sirt1 improves healthy ageing and protects from metabolic syndrome-associated cancer. Nat Commun 2010; 1: 3.

6. Lagouge M, Argmann C, Gerhart-Hines Z, Meziane H, Lerin C, Daussin F et al. Resveratrol improves mitochondrial function and protects against metabolic disease by activating SIRT1 and PGC-1alpha. Cell 2006; 127: 1109-1122.

7. Dumont M, Stack C, Elipenahli C, Jainuddin S, Launay N, Gerges M et al. PGC-1alpha overexpression exacerbates beta-amyloid and tau deposition in a transgenic mouse model of Alzheimer's disease. FASEB J 2014; 28: 1745-1755.

8. Smith MR, Syed A, Lukacsovich T, Purcell J, Barbaro BA, Worthge SA et al. A potent and selective Sirtuin 1 inhibitor alleviates pathology in multiple animal and cell models of Huntington's disease. Hum Mol Genet 2014; 23: 2995-3007.

9. Court FA, Coleman MP. Mitochondria as a central sensor for axonal degenerative stimuli. Trends Neurosci 2012; 35: 364-372.

10. Ferrer I, Aubourg P, Pujol A. General aspects and neuropathology of X-linked adrenoleukodystrophy. Brain Pathol 2010; 20: 817-830.

11. Moser H, Smith KD, Watkins PA, Powers J, Moser AB. X-linked adrenoleukodystrophy In: Scriver C (ed) The Metabolic and Molecular Bases of Inherited disease8th ednvol. II. McGraw-Hill: New-York, 2001 pp 3257-3301.

12. Engelen $M$, Barbier $M$, Dijkstra IM, Schur $R$, de Bie RM, Verhamme $C$ et al. X-linked adrenoleukodystrophy in women: a cross-sectional cohort study. Brain 2014; 137 693-706.

13. Wiesinger C, Kunze M, Regelsberger G, Forss-Petter S, Berger J. Impaired very long-chain acyl-CoA beta-oxidation in human X-linked adrenoleukodystrophy fibroblasts is a direct consequence of ABCD1 transporter dysfunction. J Biol Chem 2013; 288: 19269-19279.

14. Cartier N, Aubourg P. Hematopoietic stem cell transplantation and hematopoietic stem cel gene therapy in X-linked adrenoleukodystrophy. Brain Pathol 2010; 20: 857-862.

15. Berger J, Pujol A, Aubourg P, Forss-Petter S. Current and future pharmacological treatment strategies in X-linked adrenoleukodystrophy. Brain Pathol 2010; 20: 845-856.

16. Forss-Petter S, Werner H, Berger J, Lassmann H, Molzer B, Schwab MH et al. Targeted inactivation of the X-linked adrenoleukodystrophy gene in mice. J Neurosci Res 1997; 50: 829-843.

17. Lu JF, Lawler AM, Watkins PA, Powers JM, Moser AB, Moser HW et al. A mouse model for X-linked adrenoleukodystrophy. Proc Natl Acad Sci USA 1997; 94: 9366-9371.

18. Pujol A, Hindelang C, Callizot N, Bartsch U, Schachner M, Mandel JL. Late onset neurological phenotype of the X-ALD gene inactivation in mice: a mouse model for adrenomyeloneuropathy. Hum Mol Genet 2002; 11: 499-505

19. Pujol A, Ferrer I, Camps C, Metzger E, Hindelang C, Callizot N et al. Functional overlap between $A B C D 1$ (ALD) and ABCD2 (ALDR) transporters: a therapeutic target for X-adrenoleukodystrophy. Hum Mol Genet 2004; 13: 2997-3006.

20. Fourcade S, Lopez-Erauskin J, Galino J, Duval C, Naudi A, Jove M et al. Early oxidative damage underlying neurodegeneration in X-adrenoleukodystrophy. Hum Mol Genet 2008 17: 1762-1773.

21. Lopez-Erauskin J, Fourcade S, Galino J, Ruiz M, Schluter A, Naudi A et al. Antioxidants halt axonal degeneration in a mouse model of X-adrenoleukodystrophy. Ann Neurol 2011; 70: 84-92.

22. Galea E, Launay N, Portero-Otin M, Ruiz M, Pamplona R, Aubourg P et al. Oxidative stress underlying axonal degeneration in adrenoleukodystrophy: a paradigm for multifactorial neurodegenerative diseases? Biochim Biophys Acta 2012; 1822: 1475-1488.

23. Galino J, Ruiz M, Fourcade S, Schluter A, Lopez-Erauskin J, Guilera C et al. Oxidative damage compromises energy metabolism in the axonal degeneration mouse model of X-adrenoleukodystrophy. Antioxid Redox Signal 2011; 15: 2095-2107.

24. Morato L, Galino J, Ruiz M, Calingasan NY, Starkov AA, Dumont M et al. Pioglitazone halts axonal degeneration in a mouse model of X-linked adrenoleukodystrophy. Brain 2013; 136: 2432-2443.

25. Lopez-Erauskin J, Galino J, Ruiz M, Cuezva JM, Fabregat I, Cacabelos D et al. Impaired mitochondrial oxidative phosphorylation in the peroxisomal disease X-linked adrenoleukodystrophy. Hum Mol Genet 2013; 22: 3296-3305

26. Lopez-Erauskin J, Galino J, Bianchi P, Fourcade S, Andreu AL, Ferrer I et al. Oxidative stress modulates mitochondrial failure and cyclophilin $\mathrm{D}$ function in X-linked adrenoleukodystrophy. Brain 2012; 135: 3584-3598.

27. Fourcade S, Lopez-Erauskin J, Ruiz M, Ferrer I, Pujol A. Mitochondrial dysfunction and oxidative damage cooperatively fuel axonal degeneration in X-linked adrenoleukodystrophy. Biochimie 2014; 98: 143-149.

28. Powers JM, Pei Z, Heinzer AK, Deering R, Moser AB, Moser HW et al. Adreno-leukodystrophy: oxidative stress of mice and men. J Neuropathol Exp Neurol 2005; 64: 1067-1079.

29. Fourcade S, Ruiz M, Guilera C, Hahnen E, Brichta L, Naudi A et al. Valproic acid induces antioxidant effects in X-linked adrenoleukodystrophy. Hum Mol Genet 2010; 19: 2005-2014

30. Petrillo S, Piemonte F, Pastore A, Tozzi G, Aiello C, Pujol A et al. Glutathione imbalance in patients with X-linked adrenoleukodystrophy. Mol Genet Metab 2013; 109: 366-370.

31. Vargas CR, Wajner M, Sirtori LR, Goulart L, Chiochetta M, Coelho D et al. Evidence that oxidative stress is increased in patients with X-linked adrenoleukodystrophy. Biochim Biophys Acta 2004; 1688: 26-32.
32. Timmers S, Konings E, Bilet L, Houtkooper RH, van de Weijer T, Goossens GH et al. Calorie restriction-like effects of 30 days of resveratrol supplementation on energy metabolism and metabolic profile in obese humans. Cell Metab 2011; 14: 612-622.

33. Price NL, Gomes AP, Ling AJ, Duarte FV, Martin-Montalvo A, North BJ et al. SIRT1 is required for AMPK activation and the beneficial effects of resveratrol on mitochondrial function. Cell Metab 2012; 15: 675-690.

34. Beher D, Wu J, Cumine S, Kim KW, Lu SC, Atangan L et al. Resveratrol is not a direct activator of SIRT1 enzyme activity. Chem Biol Drug Des 2009; 74: 619-624.

35. Pfluger PT, Herranz D, Velasco-Miguel S, Serrano M, Tschop MH. Sirt1 protects against high-fat diet-induced metabolic damage. Proc Natl Acad Sci USA 2008; 105: 9793-9798.

36. Mastroeni R, Bensadoun JC, Charvin D, Aebischer P, Pujol A, Raoul C. Insulin-like growth factor-1 and neurotrophin-3 gene therapy prevents motor decline in an X-linked adrenoleukodystrophy mouse model. Ann Neurol 2009; 66: 117-122.

37. de Vries RL, Przedborski S. Mitophagy and Parkinson's disease: be eaten to stay healthy. Mol Cell Neurosci 2013; 55: 37-43.

38. Launay N, Ruiz M, Fourcade S, Schluter A, Guilera C, Ferrer I et al. Oxidative stress regulates the ubiquitin-proteasome system and immunoproteasome functioning in a mouse model of X-adrenoleukodystrophy. Brain 2013; 136: 891-904.

39. Lin MT, Beal MF. Mitochondrial dysfunction and oxidative stress in neurodegenerative diseases. Nature 2006; 443: 787-795.

40. Julien C, Tremblay C, Emond V, Lebbadi M, Salem N Jr., Bennett DA et al. Sirtuin 1 reduction parallels the accumulation of tau in Alzheimer disease. J Neuropathol Exp Neurol 2009; 68: 48-58.

41. Zhang A, Wang H, Qin X, Pang S, Yan B. Genetic analysis of SIRT1 gene promoter in sporadic Parkinson's disease. Biochem Biophys Res Commun 2012; 422: 693-696.

42. Pallas M, Pizarro JG, Gutierrez-Cuesta J, Crespo-Biel N, Alvira D, Tajes M et al. Modulation of SIRT1 expression in different neurodegenerative models and human pathologies. Neuroscience 2008; 154: 1388-1397.

43. Cui L, Jeong H, Borovecki F, Parkhurst CN, Tanese N, Krainc D. Transcriptional repression of PGC-1alpha by mutant huntingtin leads to mitochondrial dysfunction and neurodegeneration. Cell 2006; 127: 59-69.

44. Qin W, Haroutunian V, Katsel P, Cardozo CP, Ho L, Buxbaum JD et al. PGC-1alpha expression decreases in the Alzheimer disease brain as a function of dementia. Arch Neurol 2009; 66: 352-361.

45. Shin JH, Ko HS, Kang H, Lee Y, Lee YI, Pletinkova O et al. PARIS (ZNF746) repression of PGC-1alpha contributes to neurodegeneration in Parkinson's disease. Cell 2011; 144: 689-702.

46. Chen J, Zhou Y, Mueller-Steiner S, Chen LF, Kwon H, Yi S et al. SIRT1 protects against microglia-dependent amyloid-beta toxicity through inhibiting NF-kappaB signaling. J Biol Chem 2005; 280: 40364-40374.

47. Donmez G, Wang D, Cohen DE, Guarente L. SIRT1 suppresses beta-amyloid production by activating the alpha-secretase gene ADAM10. Cell 2010; 142: 320-332.

48. Kim D, Nguyen MD, Dobbin MM, Fischer A, Sananbenesi F, Rodgers JT et al. SIRT1 deacetylase protects against neurodegeneration in models for Alzheimer's disease and amyotrophic lateral sclerosis. EMBO J 2007; 26: 3169-3179.

49. Marambaud $\mathrm{P}$, Zhao H, Davies $\mathrm{P}$. Resveratrol promotes clearance of Alzheimer's disease amyloid-beta peptides. J Biol Chem 2005; 280: 37377-37382.

50. Donmez G, Arun A, Chung CY, McLean PJ, Lindquist S, Guarente L. SIRT1 protects against alpha-synuclein aggregation by activating molecular chaperones. J Neurosci 2012; 32: 124-132.

51. Jeong $H$, Cohen DE, Cui L, Supinski A, Savas JN, Mazzulli JR et al. Sirt1 mediates neuroprotection from mutant huntingtin by activation of the TORC1 and CREB transcriptional pathway. Nat Med 2012; 18: 159-165.

52. Jiang M, Wang J, Fu J, Du L, Jeong $\mathrm{H}$, West $\mathrm{T}$ et al. Neuroprotective role of Sirt1 in mammalian models of Huntington's disease through activation of multiple Sirt1 targets. Nat Med 2012; 18: 153-158.

53. Wang JT, Medress ZA, Barres BA. Axon degeneration: molecular mechanisms of a self-destruction pathway. J Cell Biol 2012; 196: 7-18.

54. Ferreirinha F, Quattrini A, Pirozzi M, Valsecchi V, Dina G, Broccoli V et al. Axonal degeneration in paraplegin-deficient mice is associated with abnormal mitochondria and impairment of axonal transport. J Clin Invest 2004; 113: 231-242.

55. Fink JK. Hereditary spastic paraplegia: clinico-pathologic features and emerging molecular mechanisms. Acta Neuropathol 2013; 126: 307-328.

56. Araki T, Sasaki Y, Milbrandt J. Increased nuclear NAD biosynthesis and SIRT1 activation prevent axonal degeneration. Science 2004; 305: 1010-1013.

57. Suzuki K, Koike T. Resveratrol abolishes resistance to axonal degeneration in slow Wallerian degeneration (WIdS) mice: activation of SIRT2, an NAD-dependent tubulin deacetylase. Biochem Biophys Res Commun 2007; 359: 665-671.

58. Wang J, Zhai Q, Chen Y, Lin E, Gu W, McBurney MW et al. A local mechanism mediates NAD-dependent protection of axon degeneration. J Cell Biol 2005; 170: 349-355.

59. Magnifico S, Saias L, Deleglise B, Duplus E, Kilinc D, Miquel MC et al. NAD+ acts on mitochondrial SirT3 to prevent axonal caspase activation and axonal degeneration. FASEBJ 2013; 27: 4712-22.

60. Adalbert R, Nogradi A, Szabo A, Coleman MP. The slow Wallerian degeneration gene in vivo protects motor axons but not their cell bodies after avulsion and neonatal axotomy. Eur $J$ Neurosci 2006; 24: 2163-2168

61. Alcendor RR, Gao S, Zhai $P$, Zablocki D, Holle E, Yu X et al. Sirt1 regulates aging and resistance to oxidative stress in the heart. Circ Res 2007; 100: 1512-1521. 
62. Liu C, Shi Z, Fan L, Zhang C, Wang K, Wang B. Resveratrol improves neuron protection and functional recovery in rat model of spinal cord injury. Brain Res 2011; 1374 100-109.

63. Rege SD, Kumar S, Wilson DN, Tamura L, Geetha T, Mathews ST et al. Resveratrol protects the brain of obese mice from oxidative damage. Oxid Med Cell Longev 2013; 2013 419092.

64. Hubbard BP, Gomes AP, Dai H, Li J, Case AW, Considine T et al. Evidence for common mechanism of SIRT1 regulation by allosteric activators. Science 2013; 339: 1216-1219.

65. Witte ME, Mahad DJ, Lassmann $\mathrm{H}$, van Horssen J. Mitochondrial dysfunction contributes to neurodegeneration in multiple sclerosis. Trends Mol Med 2014; 20: 179-187.

66. Magrane J, Sahawneh MA, Przedborski S, Estevez AG, Manfredi G. Mitochondrial dynamics and bioenergetic dysfunction is associated with synaptic alterations in mutant SOD1 motor neurons. J Neurosci 2012; 32: 229-242.
67. Ferrer I, Kapfhammer JP, Hindelang C, Kemp S, Troffer-Charlier N, Broccoli V et al. Inactivation of the peroxisomal $A B C D 2$ transporter in the mouse leads to late-onset ataxia involving mitochondria, Golgi and endoplasmic reticulum damage. Hum Mol Genet 2005; 14: 3565-3577.

68. Fourcade S, Ruiz M, Camps C, Schluter A, Houten SM, Mooyer PA et al. A key role for the peroxisomal ABCD2 transporter in fatty acid homeostasis. Am J Physiol Endocrinol Metab 2009; 296: E211-E221.

69. Liu J, Sabeva NS, Bhatnagar S, Li XA, Pujol A, Graf GA. ABCD2 is abundant in adipose tissue and opposes the accumulation of dietary erucic acid (C22:1) in fat. J Lipid Res 2010; 51: $162-168$.

70. Chabi B, Adhihetty PJ, O'Leary MF, Menzies KJ, Hood DA. Relationship between Sirt1 expression and mitochondrial proteins during conditions of chronic muscle use and disuse. J Appl Physiol (1985) 2009; 107: 1730-1735.

Supplementary Information accompanies this paper on Cell Death and Differentiation website (http://www.nature.com/cdd) 